International Journal of Applied Mathematics

Volume 34 No. $5 \quad 2021$, 901-931

ISSN: 1311-1728 (printed version); ISSN: 1314-8060 (on-line version)

doi: http://dx.doi.org/10.12732/ijam.v34i5.4

\title{
OPTIMAL CONTROL OF A POPULATION-VARYING HETEROSEXUAL HIV/AIDS MATHEMATICAL MODEL
}

\author{
Calisto Munongi ${ }^{1 \S}$, Justin M.W. Munganga ${ }^{2}$ \\ 1,2 Department of Mathematical Sciences \\ University of South Africa Science Campus \\ Florida, Unisa 0003, SOUTH AFRICA
}

\begin{abstract}
We propose a heterosexual HIV transmission model of variable population size. The model is proved to be well-posed both mathematically and epidemiologically. A threshold for the existence of the disease is established, together with the existence and asymptotic stability of equilibria. An optimal control problem is formulated in which four controls (sex education, screening, HIV prevention methods, and treatment of infectives) are administered. It is shown that there exist an optimal control set and the optimal controls are characterised by applying Pontryagin's maximum principle. We numerically solve the optimal control problem using the forward-backward sweep method and ODE45 solver. The control strategy causes an important decrease in susceptibles, unaware infectives and AIDS individuals. The strategy also causes an outstanding transfer of infectives to the HIV treatment class.
\end{abstract}

AMS Subject Classification: 92B05, 34D20, 49K15

Key Words: heterosexual HIV transmission; asymptotic stability; Lyapunov function; optimal control; Pontryagin's Maximum Principle; forward-backward sweep method

\section{Introduction}

Globally, the number of people living with HIV continue to increase (see [25],[26]). However, many persons infected with HIV are unaware of their infection. In

Received: November 20, 2020

(C) 2021 Academic Publications

${ }^{\S}$ Correspondence author 
2017, an estimated 9.2 million of the 36.9 million people did not know that they had the virus (see [26]). Furthermore, for those who are screened, access to ART remains low, especially in limited resources settings. For example, about 21.7 million people accessed ART in 2017 out of an estimated 36.9 million people living with HIV in the same year (see [26]).

Several articles have applied optimal control to specific diseases such as influenza, HIV, tuberculosis, malaria. Zhou et al. [32] study an optimal strategy for HIV multitherapy. The study establishes four types of optimal treatment strategies consisting of different combinations of reverse transcriptase inhibitors and protease inhibitors. Mallela et al. [15] develop a model that incorporates various aspects of treatments for HIV-TB co-infected individuals. Their analyses show that co-infection treatments do not influence neither the basic reproduction number nor stability of the disease-free HIV equilibria. They also suggest that, for disease eradication, single disease treatments need to be incorporated with co-infection treatments. Juusola and Brandeau [10] develop a model to assist public health decision makers ascertain how to obtain optimal investment of limited resources in HIV treatment and prevention.

There are several studies that have investigated optimal control strategies in the transmission and treatment of HIV (see [2], [6], [8],[9],[10],[11],[14],[19]). However, most of these studies have assumed symmetry in the sex structure, that is, between male and females. Moreover, some studies have considered constant population size models which are applicable to diseases where births and deaths are balanced or the time period of the disease is very short or disease-related deaths are insignificant (see [6],[9],[11],[19]). However, these assumptions fail to hold in the transmission dynamics of HIV/AIDS since the disease is endemic in humans whose communities are always of variable population sizes and the influence of disease mortality is clearly evident. For example, an estimated 940000 people died from AIDS-related illnesses globally in 2017 out of about 36.9 million people who were living with HIV (see [25]).

Our study aims to minimise HIV infection through use of targeted sex education, prevention methods, screening of unaware infectives and treatment of HIV infectives. We analyse a two-sex HIV/AIDS transmission model with a variable population size in the presence of deaths related to the disease. Optimal control is applied to obtain the most optimal intervention strategy to reduce the number of $\mathrm{HIV}$ infectives while balancing the control efforts. We define screening to include testing and counselling for HIV and other Sexually Transmitted Infections (STI's) and screening for tuberculosis (TB). Antiretrovial therapy (ART) is used to both treat HIV positive individuals and to reduce the transmission risk to an uninfected sexual partner. World health Organisa- 
tion (WHO) reports that risk of transmission to an uninfected partner by an HIV positive person who adheres to an effective ART combination can be reduced by $96 \%$ (see [25]).

HIV infection is greatly attributed to heterosexual transmission especially in Southern Africa which is the worst affected by the virus (see [26],[27]). Sex structured models may contribute meaningfully on how best to make use of limited resources in the HIV prevention, treatment and control. A gender asymmetry heterosexual model characterised by multiple concurrent partnerships is considered where rates are differentiated according to sex, e.g., susceptibility, screening, treatment and progression to AIDS (see [1],[3],[12]). In heterosexual transmission, sexual contact occurs only between partners of the opposite sex and we assume that only sexually active individuals are susceptible, that is, only horizontal HIV incidence is considered. The transmission dynamics of HIV is determined by the sexual behaviour of the infected individuals. Screening of unaware HIV infectives for treatment is important since we understand that ART reduces transmission of HIV.

In Section 2, we describe structure of heterosexual HIV/AIDS model under study. We discuss the basic properties of the model in Section 3. In Section 4, we formulate the optimal control problem, prove the existence and uniqueness of the optimal control, characterise the optimal control, and numerically compute the optimal control. Finally, conclusions are presented in Section 5.

\section{Structure of the model}

The total human population is divided into two interacting classes, namely, females $f$ and males $m$. The female and male populations are further divided into homogeneous sub-populations, namely susceptibles $S_{j}(t)$, unaware infectives $I_{1 j}(t)$, screened and aware infectives but not on treatment $I_{2 j}(t), \mathrm{HIV}$ positive individuals who are on treatment $H_{j}(t)$, and AIDS individuals $A_{j}(t)$ for $j=f, m$ where $f$ and $m$ stand for females and males respectively. We denote the total variable population sizes for females and males by $N_{f}(t)$ and $N_{m}(t)$ respectively. Here, $I_{2 j}(t), H_{j}(t)$ and $A_{j}(t)$ are new classes of infectives (with different infectivity) that arise once the disease has invaded the population. The HIV/AIDS model is as follows:

$$
\begin{aligned}
\frac{d S_{f}}{d t} & =Q_{f} N_{f}-\beta_{m f} S_{f}-\mu_{f} S_{f}, \\
\frac{d S_{m}}{d t} & =Q_{m} N_{m}-\beta_{f m} S_{m}-\mu_{m} S_{m}
\end{aligned}
$$




$$
\begin{aligned}
\frac{d I_{1 f}}{d t} & =\beta_{m f} S_{f}-\left(\theta_{f}+\delta_{f}+\mu_{f}\right) I_{1 f} \\
\frac{d I_{1 m}}{d t} & =\beta_{f m} S_{m}-\left(\theta_{m}+\delta_{m}+\mu_{m}\right) I_{1 m} \\
\frac{d I_{2 f}}{d t} & =\theta_{f} I_{1 f}-\left(\psi_{f}+\mu_{f}+\pi_{f}\right) I_{2 f} \\
\frac{d I_{2 m}}{d t} & =\theta_{m} I_{1 m}-\left(\psi_{m}+\mu_{m}+\pi_{m}\right) I_{2 m}, \\
\frac{d H_{f}}{d t} & =\pi_{f} I_{2 f}-\left(\epsilon_{f}+\mu_{f}\right) H_{f}, \\
\frac{d H_{m}}{d t} & =\pi_{m} I_{2 m}-\left(\epsilon_{m}+\mu_{m}\right) H_{m}, \\
\frac{d A_{f}}{d t} & =\delta_{f} I_{1 f}+\psi_{f} I_{2 f}+\epsilon_{f} H_{f}-\left(\mu_{f}+\alpha_{f}\right) A_{f}, \\
\frac{d A_{m}}{d t} & =\delta_{m} I_{1 m}+\psi_{m} I_{2 m}+\epsilon_{m} H_{m}-\left(\mu_{m}+\alpha_{m}\right) A_{m},
\end{aligned}
$$

with positive initial conditions while all parameters are non-negative and total population $N(t)=N_{f}(t)+N_{m}(t), N_{j}(t)=S_{j}(t)+I_{1 j}(t)+I_{2 j}(t)+H_{j}(t)+$ $A_{j}(t), j=f, m$ and $\frac{d N_{j}}{d t}=\left(Q_{j}-\mu_{j}\right) N_{j}-\alpha_{j} A_{j}$. The average number of adequate sexual contacts with female infectives per unit time of one susceptible male, $\beta_{f m}$ is given by

$$
\frac{\beta_{I_{1 f}} c_{I_{1 f}} b_{I_{1 f}} I_{1 f}+\beta_{I_{2 f}} c_{I_{2 f}} b_{I_{2 f}} I_{2 f}+\beta_{H_{f}} c_{H_{f}} b_{H_{f}} H_{f}+\beta_{A_{f}} c_{A_{f}} b_{A_{f}} A_{f}}{N_{f}} .
$$

The average number of adequate sexual contacts with male infectives per unit time of one susceptible female is given by $\beta_{m f}$ can be written in a similar way. $\beta_{m f}$ and $\beta_{f m}$ are variable functions of the total population for each sex since the population size is varying. The probability that an HIV individual will infect a susceptible depends primarily on the stage of infection and sexual behaviour of both the infective and the susceptible. Unaware infectives are considered to be in the acute HIV infection stage while aware (not on ART) infectives are in the chronic stage of the virus. Therefore we assume that $\beta_{I_{i j}}>\beta_{I_{2 j}}, j=f, m$. Furthermore, we assume that $\beta_{A_{j}}>\beta_{I_{i j}}>\beta_{I_{2 j}}>\beta_{H_{j}}$ for each $j=f, m$. The assumptions here are that individuals at the AIDS stage (if they recklessly behave) are the most infectiousness and HIV treatment reduces transmission to about $96 \%$ with good adherence (see [1],[16],[25]). $c_{j}>0$ since infectives from any class are, on average, assumed to have sexual contact with other individuals of opposite sex. The rate at which unaware infectives are detected by screening efforts depends on the size of the population of unaware infectives 
and on the prevalence of infection on each sex, that is, $\theta_{j}=\theta_{j}\left(I_{1 j}, N_{j}\right)$. The AIDS individuals are assumed to be capable of transmitting the virus. Utilising our model, we can define the total number of new HIV infections, $I_{\text {new }}$, and disease-related deaths, $A_{D}$, as follows:

$$
\begin{array}{r}
I_{\text {new }}=\int_{0}^{T}\left(\beta_{m f} S_{f}+\beta_{f m} S_{m}\right) d t, \\
A_{D}=\int_{0}^{T}\left(\alpha_{f} A_{f}+\alpha_{m} A_{m}\right) d t,
\end{array}
$$

where $T$ is the final time. The total HIV and AIDS burden can be represented by $k_{1} I_{\text {new }}+k_{2} A_{D}$ where $k_{1}$ and $k_{2}$ are weighted coefficients delegated to new HIV infections and disease-related deaths, respectively. New HIV infections and disease-related deaths have great importance in the effort to reduce the effects of HIV as a world epidemic (see [27]). However, there are several other expressions for the total burden of HIV and AIDS depending on the priorities of the studies (see [15]). The meaning of our parameters is shown in Table 1.

\section{Basic properties of the model}

\subsection{Well-posedness of the model}

Our interest is on states that are non-negative. It is elementary to show that non-negative initial conditions to the model (1a)-(1j) lead to non-negative solutions for all time $t \geq 0$ and will remain non-negative. Therefore the mathematically and epidemiologically feasible region for system (1a)-(1j) is given by

$$
D=\left\{\begin{array}{l|l}
\mathbf{X} \in \mathbf{R}_{+}^{10} & \begin{array}{c}
S_{f}+I_{1 f}+I_{2 f}+H_{f}+A_{f}=N_{f} \\
S_{m}+I_{1 m}+I_{2 m}+H_{m}+A_{m}=N_{m}
\end{array}
\end{array}\right\},
$$

where $\mathbf{X}=\left(S_{f}, S_{m}, I_{1 f}, I_{1 m}, I_{2 f}, I_{2 m}, H_{f}, H_{m}, A_{f}, A_{m}\right)$.

\subsection{Constant population size}

The total populations $N_{j}(t), j=f, m$ may remain constant when $\frac{d N_{j}}{d t}=0$, that is, when $\left(Q_{j}-\mu_{j}\right) N_{j}-\alpha_{j} A_{j}=0$. In this case, the number of AIDS individuals cannot be identically zero and the following restrictions are imposed: $Q_{f}=\mu_{f}, Q_{m}=\mu_{m}$ and $\alpha_{j}=0 \forall j=f, m$. Our interest in this paper is to study the model when population is not constant and we assume that $Q_{j} \neq \mu_{j}$ 
Table 1: Description of parameters (all non-negative); $j=f, m$

\begin{tabular}{|l|l|}
\hline Parameter & Symbol \\
\hline $\begin{array}{l}\text { Probability of transmitting HIV by unaware } \\
\text { infective to a susceptible during sexual encounter }\end{array}$ & $\beta_{I_{1 j}}$ \\
\hline $\begin{array}{l}\text { Probability of transmitting HIV by aware infective } \\
\text { not on ART during sexual encounter }\end{array}$ & $\beta_{I_{2 j}}$ \\
\hline $\begin{array}{l}\text { Probability of transmitting HIV by infective on } \\
\text { ART during sexual encounter }\end{array}$ & $\beta_{H_{j}}$ \\
\hline $\begin{array}{l}\text { Probability of transmitting HIV by AIDS } \\
\text { individual during sexual encounter }\end{array}$ & $\beta_{A_{j}}$ \\
\hline Per capita growth rate at sexual maturity age & $Q_{j}$ \\
\hline Average number of partners of unaware infectives & $c_{I_{1 j}}$ \\
\hline Average number of partners of aware infectives & $c_{I_{2 j}}$ \\
\hline Average number of partners of infectives on ART & $c_{H_{j}}$ \\
\hline Average number of sexual partners of AIDS persons & $c_{A_{j}}$ \\
\hline $\begin{array}{l}\text { Probability of unaware infective to have sexual } \\
\text { encounter with a susceptible of opposite sex }\end{array}$ & $b_{I_{1 j}}$ \\
\hline $\begin{array}{l}\text { Probability of aware infective to have sexual } \\
\text { encounter with a susceptible of opposite sex }\end{array}$ & $b_{I_{2 j}}$ \\
\hline $\begin{array}{l}\text { Probability of infective on ART to have sexual } \\
\text { encounter with a susceptible of opposite sex }\end{array}$ & $b_{H_{j}}$ \\
\hline $\begin{array}{l}\text { Probability of index partner with AIDS to have } \\
\text { sexual encounter with a susceptible of opposite sex }\end{array}$ & $b_{A_{j}}$ \\
\hline Rate at which unaware infectives are screened & $\theta A_{j}$ \\
\hline Rate at which unaware infective progress to AIDS & $\delta_{j}$ \\
\hline Progression to AIDS by aware infective not on ART & $\psi_{j}$ \\
\hline Rate of treatment for screened and aware infective & $\pi_{j}$ \\
\hline Rate at which infective on ART progress to AIDS & $\epsilon_{j}$ \\
\hline Per capita disease-free death rate & $\mu_{j}$ \\
\hline Disease-related death rate for AIDS individuals & $\alpha_{j}$ \\
\hline
\end{tabular}


for any $j=f, m$ and each of $\alpha_{j} \neq 0$. In order to bring the disease under control, we require that the number of infectives $\left(I_{1 j} \rightarrow 0\right)$. However, this may be problematic if the total population size is varying. For example, if the total population grows faster than the infectives, then the number of infectives could grow abundantly although the proportion $i_{j} \rightarrow 0$. It is therefore essential to make use of proportions of the sub-populations.

\subsection{Varying population size}

Based on the system (1a)-(1j), we introduce proportions of individuals in the epidemiological classes as follows:

$$
s_{f}=\frac{S_{f}}{N_{f}}, i_{1 f}=\frac{I_{1 f}}{N_{f}}, i_{2 f}=\frac{I_{2 f}}{N_{f}}, h_{f}=\frac{H_{f}}{N_{f}}, a_{f}=\frac{A_{f}}{N_{f}} .
$$

Proportions for males are done in a similar manner. $\beta_{m f}$ and $\beta_{f m}$ can now be written as:

$$
\beta_{I_{1 m}} c_{I_{1 m}} b_{I_{1 m}} i_{1 m}+\beta_{I_{2 m}} c_{I_{2 m}} b_{I_{2 m}} i_{2 m}+\beta_{H_{m}} c_{H_{m}} b_{H_{m}} h_{m}+\beta_{A_{m}} c_{A_{m}} b_{A_{m}} a_{m}
$$

and

$$
\beta_{I_{1 f}} c_{I_{1 f}} b_{I_{1 f}} i_{1 f}+\beta_{I_{2 f}} c_{I_{2 f}} b_{I_{2 f}} i_{2 f}+\beta_{H_{f}} c_{H_{f}} b_{H_{f}} h_{f}+\beta_{A_{f}} c_{A_{f}} b_{A_{f}} a_{f},
$$

respectively. The resulting system of differential equations is

$$
\begin{aligned}
\frac{d s_{f}}{d t} & =Q_{f}-Q_{f} s_{f}-\beta_{m f} s_{f}+\alpha_{f} a_{f} s_{f}, \\
\frac{d s_{m}}{d t} & =Q_{m}-Q_{m} s_{m}-\beta_{f m} s_{m}+\alpha_{m} a_{m} s_{m}, \\
\frac{d i_{1 f}}{d t} & =\beta_{m f} s_{f}-\left(Q_{f}+\theta_{f}+\delta_{f}\right) i_{1 f}+\alpha_{f} a_{f} i_{1 f}, \\
\frac{d i_{1 m}}{d t} & =\beta_{f m} s_{m}-\left(Q_{m}+\theta_{m}+\delta_{m}\right) i_{1 m}+\alpha_{m} a_{m} i_{1 m}, \\
\frac{d i_{2 f}}{d t} & =\theta_{f} i_{1 f}-\left(Q_{f}+\psi_{f}+\pi_{f}\right) i_{2 f}+\alpha_{f} a_{f} i_{2 f}, \\
\frac{d i_{2 m}}{d t} & =\theta_{m} i_{1 m}-\left(Q_{m}+\psi_{m}+\pi_{m}\right) i_{2 m}+\alpha_{m} a_{m} i_{2 m}, \\
\frac{d h_{f}}{d t} & =\pi_{f} i_{2 f}-\left(Q_{f}+\epsilon_{f}\right) h_{f}+\alpha_{f} h_{f} a_{f}, \\
\frac{d h_{m}}{d t} & =\pi_{m} i_{2 m}-\left(Q_{m}+\epsilon_{m}\right) h_{m}+\alpha_{m} h_{m} a_{m},
\end{aligned}
$$




$$
\begin{aligned}
\frac{d a_{f}}{d t} & =\delta_{f} i_{1 f}+\psi_{f} i_{2 f}+\epsilon_{f} h_{f}-\left(Q_{f}+\alpha_{f}\right) a_{f}+\alpha_{f} a_{f}^{2} \\
\frac{d a_{m}}{d t} & =\delta_{m} i_{1 m}+\psi_{m} i_{2 m}+\epsilon_{m} h_{m}-\left(Q_{m}+\alpha_{m}\right) a_{m}+\alpha_{m} a_{m}^{2}
\end{aligned}
$$

where

$$
\begin{aligned}
\frac{d n_{f}}{d t} & =Q_{f}\left(1-n_{f}\right)+\alpha_{f} a_{f}\left(n_{f}-1\right), \\
\frac{d n_{m}}{d t} & =Q_{m}\left(1-n_{m}\right)+\alpha_{m} a_{m}\left(n_{m}-1\right) .
\end{aligned}
$$

If we assume that $n_{i}(0) \leq 1, i=f, m$, then it follows from equations (4a) and (4b) that $\frac{d n_{i}}{d t} \leq Q_{i}\left(1-n_{i}\right)$. Therefore we conclude that $n_{i}(t) \leq 1$ for $i=f, m$ and a biologically feasible region for the model $(3 \mathrm{a}-3 \mathrm{j})$ is given by

$$
\begin{gathered}
\Omega_{1}=\left\{\mathbf{x} \mid \begin{array}{c}
s_{f}, i_{1 f}, i_{2 f}, h_{f}, a_{f} \geq 0, n_{f} \leq 1 \\
s_{m}, i_{1 m}, i_{2 m}, h_{m}, a_{m} \geq 0, n_{m} \leq 1
\end{array}\right\} \\
\mathbf{x}=\left(s_{f}, s_{m}, i_{1 f}, i_{1 m}, i_{2 f}, i_{2 m}, h_{f}, h_{m}, a_{f}, a_{m}\right)
\end{gathered}
$$

and

$$
n_{j}=s_{j}+i_{1 j}+i_{2 j}+h_{j}+a_{j}, \quad j=f, m .
$$

Theorem 1. The system (3a-3j) always has a disease free equilibrium point (DFE) given by $E^{0}=(1,1,0,0,0,0,0,0,0,0)$.

Proof. Using the substitutions $s_{f}=1-i_{1 f}-i_{2 f}-h_{f}-a_{f}$ and $s_{m}=1-i_{1 m}-i_{2 m}-h_{m}-a_{m}$, equations (3c) and (3d) become

$$
\begin{aligned}
\frac{d i_{1 f}}{d t} & =\beta_{m f}\left(1-i_{1 f}-i_{2 f}-h_{f}-a_{f}\right)-\left(Q_{f}+\theta_{f}+\delta_{f}\right) i_{1 f} \\
& +\alpha_{f} a_{f} i_{1 f} \\
\frac{d i_{1 m}}{d t} & =\beta_{f m}\left(1-i_{1 m}-i_{2 m}-h_{m}-a_{m}\right)-\left(Q_{m}+\theta_{m}+\delta_{m}\right) i_{1 m} \\
& +\alpha_{m} a_{m} i_{1 m} .
\end{aligned}
$$

Equations (6a) and (6b), together with equations (3e-3j) form a system in the $i_{1 f}, i_{1 m}, i_{2 f}, i_{2 m}, h_{f}, h_{m}, a_{f}, a_{m}$ region $\Omega_{2}$ given by

$$
\Omega_{2}=\left\{\left(i_{1 f}, i_{1 m}, i_{2 f}, i_{2 m}, h_{f}, h_{m}, a_{f}, a_{m}\right) \mid\right.
$$




$$
\left.\begin{array}{c}
i_{1 f}, i_{2 f}, h_{f}, a_{f} \geq 0, i_{1 f}+i_{2 f}+h_{f}+a_{f} \leq 1 \\
i_{1 m}, i_{2 m}, h_{m}, a_{m} \geq 0, i_{1 m}+i_{2 m}+h_{m}+a_{m} \leq 1
\end{array}\right\}
$$

Noting that equilibrium points of the system are obtained by solving $\frac{d s_{f}}{d t}=\frac{d s_{m}}{d t}=\frac{d i_{1 f}}{d t}=\frac{d i_{1 m}}{d t}=\frac{d i_{2 f}}{d t}=\frac{d i_{2 m}}{d t}=\frac{d h_{f}}{d t}=\frac{d h_{m}}{d t}=\frac{d a_{f}}{d t}=\frac{d a_{m}}{d t}=0$, it is clear that if $i_{1 f}=i_{1 m}=0$ then $i_{2 f}=1_{2 m}=h_{f}=h_{m}=a_{f}=a_{m}=0$ implying that $s_{f}=s_{m}=1$. Therefore the DFE $E^{0}$ always exists and is unique.

\subsubsection{Existence of a threshold parameter}

The reproduction number, denoted $\mathcal{R}_{0}$ is defined as the expected number of secondary cases produced, in a completely susceptible population, by a typical infected individual during its entire period of infectiousness (see [5]). The HIV/AIDS transmission model (3a)-(3h) comprises of nonnegative initial conditions and a system of equations of the form: $\dot{x}=f_{i}(x)=\mathcal{F}_{i}(x)-$ $\mathcal{V}_{i}(x), i=1,2, \cdots, n$ where the functions and $\mathcal{V}_{i}=\mathcal{V}_{i}^{-}-\mathcal{V}_{i}^{+}$satisfy assumptions $\left(A_{1}\right)-\left(A_{5}\right)$ in [28]. We use linear stability of the next generation operator method to find $\mathcal{R}_{0}$, the spectral radius of the matrix $F V^{-1}$. After some calculations, we get $\mathcal{R}_{0}=\sqrt{\mathcal{R}_{f} \mathcal{R}_{m}}$. For $j=f, m$; we have

$$
\begin{aligned}
\mathcal{R}_{j} & =\frac{\beta_{I_{1 j}} c_{I_{1 j}} b_{I_{1 j}}}{Q_{j}+\theta_{j}+\delta_{j}}+\frac{\beta_{I_{2 j}} c_{I_{2 j}} b_{I_{2 j}} \theta_{j}}{\left(Q_{j}+\theta_{j}+\delta_{j}\right)\left(Q_{j}+\psi_{j}+\pi_{j}\right)} \\
& +\frac{\beta_{H_{j}} c_{H_{j}} b_{H_{j}} \theta_{j} \pi_{j}}{\left(Q_{j}+\epsilon_{j}\right)\left(Q_{j}+\theta_{j}+\delta_{j}\right)\left(Q_{j}+\psi_{j}+\pi_{j}\right)} \\
& +\frac{\beta_{A_{j}} c_{A_{j}} b_{A_{j}}\left(\theta_{j} \pi_{j} \epsilon_{j}+\left(Q_{j}+\epsilon_{j}\right)\left(\delta_{j}\left(Q_{j}+\psi_{j}+\pi_{j}\right)+\theta_{j} \psi_{j}\right)\right)}{\left(Q_{j}+\alpha_{j}\right)\left(Q_{j}+\epsilon_{j}\right)\left(Q_{j}+\theta_{j}+\delta_{j}\right)\left(Q_{j}+\psi_{j}+\pi_{j}\right)}
\end{aligned}
$$

$\mathcal{R}_{0}$ is the geometric mean of reproduction number for females $\left(\mathcal{R}_{f}\right)$ and that for males $\left(\mathcal{R}_{m}\right)$. $\mathcal{R}_{0}$ take the above form since, in heterosexual transmission, the cycle for infection for each sex must go through the opposite sex, that is, male-female-male and female-male-female. Each of the parameters used in the model (3a)-(3h) do appear in the expression of $\mathcal{R}_{0}$, showing that they have some influence on the asymptotic tendency of the total population size. The structures of $R_{f}$ and $R_{m}$ suggest that they represent sums of contact numbers of the 4 infective classes for females and males, respectively. Therefore $R_{0}$ has the accurate conception that it is the expected number of secondary cases produced, in a completely susceptible population, by a typical infected individual during its entire period of infectiousness. The first term in $R_{f}$ is the contact number for 
unaware female infective and it is the product of the contact rate $\beta_{I_{1 f}} c_{I_{1 f}} b_{I_{1 f}}$ of unaware infectives per unit time and the average duration spent in that class adapted for female population growth of $1 /\left(Q_{f}+\theta_{f}+\delta_{f}\right)$. The second term in $R_{f}$ is the product of the contact rate $\beta_{h_{f}} c_{h_{f}} b_{h_{f}}$ of HIV individuals on treatment, the average infectious period spent in this class $1 /\left(Q_{f}+\psi_{f}+\pi_{f}\right)$ and the fraction $\theta_{f} /\left(Q_{f}+\theta_{f}+\delta_{f}\right)$ of female infectives surviving the unaware class. The contact number for AIDS individual (fourth term in $R_{f}$ ) is the product of AIDS individuals' contact rate $\beta_{a_{f}} c_{a_{f}} b_{a_{f}}$, the average infectious period spent in this class adapted for for female population growth $1 /\left(Q_{f}+\alpha_{f}\right)$ and the fraction $\frac{\left(\theta_{f} \pi_{f} \epsilon_{f}+\left(Q_{f}+\epsilon_{f}\right)\left(\delta_{f}\left(Q_{f}+\psi_{f}+\pi_{f}\right)+\theta_{f} \psi_{f}\right)\right)}{\left(Q_{f}+\epsilon_{f}\right)\left(Q_{f}+\theta_{f}+\delta_{f}\right)\left(Q_{f}+\psi_{f}+\pi_{f}\right)}$ surviving the three earlier infective stages (unaware, aware and treatment). It is possible to have in the class of AIDS individuals, a fraction that survives treatment, because of imperfect adherence (due to side effects, medication fatigue), drug resistance mutations and inadequate sustainable programs for accessing ART in limited resource settings (see [18],[20],[23]).

Theorem 2. The DFE $E^{0}=(1,1,0,0,0,0,0,0,0,0)$ is locally asymptotically stable in $\Omega_{1}$ if $\mathcal{R}_{0}<1$, and unstable if $\mathcal{R}_{0}>1$.

Proof. We use the Jacobian of the system (3a-3j) to establish the local stability of $E_{0}$. The first two eigenvalues of $J_{E_{0}}$ are $\lambda_{1}=-Q_{f}<0, \lambda_{2}=$ $-Q_{m}<0$. The remaining eight eigenvalues are obtained from the block matrix

$$
\begin{aligned}
& J_{1}=\left(\begin{array}{cccccccc}
-a & B_{1 m} & 0 & B_{2 m} & 0 & B_{3 m} & 0 & B_{4 m} \\
B_{1 f} & -p & B_{2 f} & 0 & B_{3 f} & 0 & B_{4 f} & 0 \\
\theta_{f} & 0 & -d & 0 & 0 & 0 & 0 & 0 \\
0 & \theta_{m} & 0 & -q & 0 & 0 & 0 & 0 \\
0 & 0 & \pi_{f} & 0 & -g & 0 & 0 & 0 \\
0 & 0 & 0 & \pi_{m} & 0 & -r & 0 & 0 \\
\delta_{f} & 0 & \psi_{f} & 0 & \epsilon_{f} & 0 & -h & 0 \\
0 & \delta_{m} & 0 & \psi_{m} & 0 & \epsilon_{m} & 0 & -s \\
& & & & & & &
\end{array}\right) \\
& B_{1 m}=\beta_{I_{1 m}} c_{I_{1 m}} b_{I_{1 m}}, B_{2 m}=\beta_{I_{2 m}} c_{I_{2 m}} b_{I_{2 m}}, B_{3 m}=\beta_{H_{m}} c_{H_{m}} b_{H_{m}}, \\
& B_{4 m}=\beta_{A_{m}} c_{A_{m}} b_{A_{m}}, B_{1 f}=\beta_{I_{1 f}} c_{I_{1 f}} b_{I_{1 f}}, B_{2 f}=\beta_{I_{2 f}} c_{I_{2 f}} b_{I_{2 f}}, \\
& B_{3 f}=\beta_{H_{f}} c_{H_{f}} b_{H_{f}}, B_{4 f}=\beta_{A_{f}} c_{A_{f}} b_{A_{f}}, \\
& a=Q_{f}+\theta_{f}+\delta_{f}, d=Q_{f}+\psi_{f}+\pi_{F}, g=Q_{f}+\epsilon_{f}, h=Q_{f}+\alpha_{f},
\end{aligned}
$$




$$
p=Q_{m}+\theta_{m}+\delta_{m}, q=Q_{m}+\psi_{m}+\pi_{m}, r=Q_{m}+\epsilon_{m}, s=Q_{m}+\alpha_{m} .
$$

The trace and determinant of $J_{1}$ are given by:

$$
\begin{aligned}
& \operatorname{tr}\left(J_{1}\right)=-(a+p+d+q+g+r+h+s)<0 \\
& \operatorname{det}\left(J_{1}\right)=A_{1}+A_{9}-\left(A_{2}+A_{3}+A_{4}+A_{5}+A_{6}+A_{7}\right) \\
& \quad=\left(2 A_{1}+A_{8}+A_{9}\right)\left(1-\mathcal{R}_{0}^{2}\right)+\left(\frac{2 A_{1}+A_{8}+A_{9}}{A_{9}}-1\right) \sum_{i=1}^{8} A_{i}
\end{aligned}
$$

where

$$
\begin{aligned}
A_{1} & =B_{1 f} \theta_{m}\left(B_{2 m} r+B_{3 m} \pi_{m}\right) d g h s+B_{1 f} B_{4 m} \theta_{m} d g h\left(\pi_{m} \epsilon_{m}\right. \\
& \left.+\psi_{m} r\right)+B_{3 f} B_{1 m} \theta_{f} \pi_{f} h q r s+B_{4 f} B_{1 m} \theta_{f} \pi_{f} \epsilon_{f} q r s \\
A_{2} & =B_{3 f} B_{3 m} \theta_{f} \theta_{m} \pi_{f} \pi_{m} h s+B_{4 m} \theta_{f} \theta_{m} \pi_{f} \pi_{m} \epsilon_{m}\left(B_{3 f} h+B_{4 f} \epsilon_{f}\right) \\
& +B_{2 m} \theta_{f} \theta_{m} \pi_{f} r s\left(B_{3 f} h+B_{4 f} \epsilon_{f}\right) \\
A_{3} & =B_{4 m} \theta_{f} \theta_{m} \pi_{f} \psi_{m} r\left(B_{3 f} h+B_{4 f} \epsilon_{f}\right)+B_{2 f} \theta_{f} \theta_{m} \pi_{m} g h\left(B_{3 m} s\right. \\
& \left.+B_{4 m} \epsilon_{m}\right) \\
A_{4} & =B_{4 f} \theta_{f} \theta_{m} \pi_{m} \psi_{f} g\left(B_{3 m} s+B_{4 m} \epsilon_{m}\right)+B_{2 m} \theta_{f} \theta_{m} g r s\left(B_{2 f} h\right. \\
& \left.+B_{4 f} \psi_{f}\right)+B_{4 m} \theta_{f} \theta_{m} \psi_{m} g r\left(B_{2 f} h+B_{4 f} \psi_{f}\right) \\
A_{5} & =B_{4 m} \theta_{f} \pi_{f} \delta_{m} r q\left(B_{3 f} h+B_{4 f} \epsilon_{f}\right)+B_{1 m} \theta_{f} g r s q\left(B_{2 f} h\right. \\
& \left.+B_{4 f} \psi_{f}\right)+B_{4 m} \theta_{f} \delta_{m} g r q\left(B_{2 f} h+B_{4 f} \psi_{f}\right) \\
A_{6} & =B_{4 f} \theta_{m} \delta_{f} \pi_{m} g d\left(B_{3 m} s+B_{4 m} \epsilon_{m}\right)+B_{4 f} \theta_{m} \delta_{f} g r d\left(B_{2 m} s\right. \\
& \left.+B_{4 m} \psi_{m}\right)+B_{1 m} B_{4 f} \delta_{f} g r d q s \\
A_{7} & =B_{4 f} B_{4 m} \delta_{f} \delta_{m} g r d q+B_{1 f} g r h d q\left(B_{1 m} s+B_{4 m} \delta_{m}\right) \\
A_{8} & =B_{4 f} B_{3 m} \theta_{f} \theta_{m} \pi_{f} \pi_{m} \epsilon_{f} s \\
A_{9} & =a d g h p q r s
\end{aligned}
$$

so that $\mathcal{R}_{0}^{2}=\frac{\left(A_{1}+A_{2}+A_{3}+A_{4}+A_{5}+A_{6}+A_{7}+A_{8}\right.}{A_{9}}=R_{f} R_{m}$. Since the second term in the expression $\operatorname{det}\left(J_{1}\right)$ is always positive, then $\operatorname{det}\left(J_{1}\right)$ is positive if $\mathcal{R}_{0}<1$. Therefore, $\lambda_{i}<0$ for $i=3,4, \cdots, 10$. Thus $E_{0}$ is locally asymptotically stable if and only if $\mathcal{R}_{0}<1$. If $\mathcal{R}_{0}>1$, then the first term of $\operatorname{det}\left(J_{1}\right)$ is positive and $\operatorname{det}\left(J_{1}\right)$ is positive since its second is always positive. Therefore, the DFE is unstable if $\mathcal{R}_{0}>1$.

Theorem 3. If $\mathcal{R}_{0}<1$, then the DFE $E^{0}$ is globally asymptotically stable $(G A S)$ in $\Omega_{1}$ and is unstable if $\mathcal{R}_{0}>1$. 
Proof. The global asymptotic stability of the DFE is established by the existence of a Lyapunov function involving the Perron eigenvector (see [22]). According to Theorem 2.1 in [22], for the model (3a-3j) in $\Omega_{1}$, there exist a Lyapunov function given by $Q=w^{T} V^{-1} x$ where $w$ is the Perron eigenvector, that is, the left eigenvector of the nonnegative matrix $V^{-1} F$ corresponding to the eigenvalue $\rho\left(F V^{-1}\right)=\mathcal{R}_{0}$. Here, $x=\left(i_{1 f}, i_{1 m}, i_{2 f}, i_{2 m}, h_{f}, h_{m}, a_{f}, a_{m}\right)^{T}$, $y=\left(s_{f}, s_{m}\right)$, and $F, V, V^{-1}$ are calculated in Theorem 1:

$$
\begin{aligned}
f(x, y)= & (F-V) x-\mathcal{F}(x, y)+\mathcal{V}(x, y) \\
= & \left(\begin{array}{c}
\beta_{m f}\left(1-s_{f}\right)+\alpha_{f} a_{f} i_{1 f} \\
\beta_{f m}\left(1-s_{m}\right)+\alpha_{m} a_{m} i_{1 m f} \\
\alpha_{f} a_{f} i_{2 f} \\
\alpha_{m} a_{m} i_{2 m} \\
\alpha_{f} a_{f} h_{f} \\
\alpha_{m} a_{m} h_{m} \\
\alpha_{f} a_{f}^{2} \\
\alpha_{m} a_{m}^{2}
\end{array}\right) .
\end{aligned}
$$

We note that $F \geq 0, V^{-1} \geq 0, f(x, y) \geq 0$ and let $w^{T}=\left(v_{1}, v_{2}, v_{3}, v_{4}, v_{5}, v_{6}, v_{7}, v_{8}\right)$ be the left eigenvector corresponding to the eigenvalue $\rho\left(V^{-1} F\right)$. Then $w^{T}\left(V^{-1} F\right)=\left(\rho\left(V^{-1} F\right)\right) w^{T}$ such that $\left(\rho\left(V^{-1} F\right)\right) w^{T}=$ $\sqrt{\frac{B_{4 m} k_{f}}{a d g h} \frac{B_{4 f} k_{m}}{p q r s}} w^{T}$, where

$k_{m}=r\left(q \delta_{m}+\theta_{m} \psi_{m}\right)+\theta_{m} \pi_{m} \epsilon_{m}$ and $k_{f}=g\left(d \delta_{f}+\theta_{f} \psi_{f}\right)+\theta_{f} \pi_{f} \epsilon_{f}$. Therefore, $w^{T}=\left(0,0,0,0,0,0,0, v_{7}, v_{8}\right)$. By Theorem 2.1 in [22], $Q=w^{T} V^{-1} x$ is the Lyapunov function for the system $(3 \mathrm{a})-(3 \mathrm{j})$ when $\mathcal{R}_{0}<1$ and

$$
\begin{aligned}
Q & =\left(\frac{k_{f} i_{1 f}}{a d g h}+\frac{g \psi_{f}+\pi_{f} \epsilon_{f} i_{2 f}}{d g h}+\frac{\epsilon_{f} h_{f}}{g h}+\frac{a_{f}}{h}\right) v_{7} \\
& +\left(\frac{k_{m} i_{1 m}}{p q r s}+\frac{r \psi_{m}+\pi_{m} \epsilon_{m} i_{2 m}}{q r s}+\frac{\epsilon_{m} h_{m}}{r s}+\frac{a_{m}}{s}\right) v_{8} .
\end{aligned}
$$

\subsubsection{Existence and uniqueness of endemic equilibrium}

The establishment of the endemic equilibrium is confined to the case $\mathcal{R}_{0}>1$. Letting $\mathbf{x}=\left(s_{f}, s_{m}, i_{1 f}, i_{1 m}, i_{2 f}, i_{2 m}, h_{f}, h_{m}, a_{f}, a_{m}\right)$, the endemic equilibrium point $E_{1}: \mathbf{x}=\mathbf{x}^{\star} \in \Omega_{1}$, is obtained by equating to zero the right hand sides 
of the system $(3 \mathrm{a}-3 \mathbf{j})$ together with $\mathbf{x}^{\star}>0$. Adding the right hand sides of the equations $(3 \mathrm{a}),(3 \mathrm{c}),(3 \mathrm{e}),(3 \mathrm{~g}),(3 \mathrm{i})$ leads to

$$
Q_{f}\left(1-s_{f}^{\star}-i_{1 f}^{\star}-i_{2 f}^{\star}-h_{f}^{\star}-a_{f}^{\star}\right)-\alpha_{f} a_{f}^{\star}\left(1-s_{f}^{\star}-i_{1 f}^{\star}-i_{2 f}^{\star}-h_{f}^{\star}-a_{f}^{\star}\right)=0 .
$$

Therefore, $0<a_{f}^{\star}<\min \left\{Q_{f} / \alpha_{f}, 1\right\}$ and $0<a_{m}^{\star}<\min \left\{Q_{m} / \alpha_{m}, 1\right\}$. The coordinates of the endemic equilibrium $E_{1}$ as are given by:

$$
\begin{aligned}
& i_{1 f}^{\star}=\frac{Y_{f}}{\theta_{f} \pi_{f} a_{f}^{\star}\left(h-\alpha_{f} a_{f}^{\star}\right)}\left(1-a_{f}^{\star}-\frac{Y_{f}}{Z_{f}}\left(1+\frac{\theta_{f}}{\left(d-\alpha_{f} a_{f}^{\star}\right)}\right.\right. \\
& \left.\left.+\frac{Z_{m}}{X_{m}}\left(a-\alpha_{f} a_{f}^{\star}\right)\right)\right), \\
& i_{1 m}^{\star}=\frac{Y_{m}}{\theta_{m} \pi_{m} a_{m}^{\star}\left(s-\alpha_{m} a_{m}^{\star}\right)}\left(1-a_{m}^{\star}-\frac{Y_{m}}{Z_{m}}\left(1 \frac{\theta_{m}}{\left(q-\alpha_{m} a_{m}^{\star}\right)}\right.\right. \\
& \left.\left.+\frac{Z_{f}}{X_{f}}\left(p-\alpha_{m} a_{m}^{\star}\right)\right)\right), \\
& i_{2 f}^{\star}=\frac{g-\alpha_{f} a_{f}^{\star}}{\pi_{f}}\left(1-a_{f}^{\star}-\frac{Y_{f}}{Z_{f}}\left(1+\frac{\theta_{f}}{\left(d-\alpha_{f} a_{f}^{\star}\right)}\right.\right. \\
& \left.\left.+\frac{Z_{m}}{X_{m}}\left(a-\alpha_{f} a_{f}^{\star}\right)\right)\right) \\
& i_{2 m}^{\star}=\frac{r-\alpha_{m} a_{m}^{\star}}{\pi_{m}}\left(1-a_{m}^{\star}-\frac{Y_{m}}{Z_{m}}\left(1+\frac{\theta_{m}}{\left(q-\alpha_{m} a_{m}^{\star}\right)}\right.\right. \\
& \left.\left.+\frac{Z_{f}}{X_{f}}\left(p-\alpha_{m} a_{m}^{\star}\right)\right)\right) \\
& h_{f}^{\star}=1-a_{f}^{\star}-\frac{Y_{f}}{Z_{f}}\left(1+\frac{\theta_{f}}{\left(d-\alpha_{f} a_{f}^{\star}\right)}+\frac{Z_{m}}{X_{m}}\left(a-\alpha_{f} a_{f}^{\star}\right)\right), \\
& h_{m}^{\star}=1-a_{m}^{\star}-\frac{Y_{m}}{Z_{m}}\left(1+\frac{\theta_{m}}{\left(q-\alpha_{m} a_{m}^{\star}\right)}+\frac{Z_{f}}{X_{f}}\left(p-\alpha_{m} a_{m}^{\star}\right)\right) \text {, }
\end{aligned}
$$

with $s_{f}^{\star}=1-i_{1 f}^{\star}-i_{2 f}^{\star}-h_{f}^{\star}-a_{f}^{\star}, s_{m}^{\star}=1-i_{1 m}^{\star}-i_{2 m}^{\star}-h_{m}^{\star}-a_{m}^{\star}$,

$$
\begin{aligned}
& X_{f}=B_{1 f} Y_{f}+B_{2 f} \frac{\theta_{f} Y_{f}}{d-\alpha_{f} a_{f}^{\star}}+B_{3 f} \theta_{f} \pi_{f} a_{f}^{\star}\left(h-\alpha_{f} a_{f}^{\star}\right)+B_{4 f} a_{f}^{\star} Z_{f}, \\
& X_{m}=B_{1 m} Y_{m}+B_{2 m} \frac{\theta_{m} Y_{m}}{q-\alpha_{m} a_{m}^{\star}}+B_{3 m} \theta_{m} \pi_{m} a_{m}^{\star}\left(s-\alpha_{m} a_{m}^{\star}\right)+B_{4 m} a_{m}^{\star} Z_{m}, \\
& Y_{f}=a_{f}^{\star}\left(g-\alpha_{f} a_{f}^{\star}\right)\left(h-\alpha_{f} a_{f}^{\star}\right)\left(d-\alpha_{f} a_{f}^{\star}\right), \\
& Y_{m}=a_{m}^{\star}\left(r-\alpha_{m} a_{m}^{\star}\right)\left(s-\alpha_{m} a_{m}^{\star}\right)\left(q-\alpha_{m} a_{m}^{\star}\right), \\
& Z_{f}=\theta_{f} \pi_{f}\left(\delta_{f}\left(d-\alpha_{f} a_{f}^{\star}\right)\left(g-\alpha_{f} a_{f}^{\star}\right)+\theta_{f} \psi_{f}\left(g-\alpha_{f} a_{f}^{\star}\right)+\theta_{f} \pi_{f} \epsilon_{f}\right), \\
& Z_{m}=\theta_{m} \pi_{m}\left(\delta_{m}\left(q-\alpha_{m} a_{m}^{\star}\right)\left(r-\alpha_{m} a_{m}^{\star}\right)+\theta_{m} \psi_{m}\left(r-\alpha_{m} a_{m}^{\star}\right)+\theta_{m} \pi_{m} \epsilon_{m}\right) .
\end{aligned}
$$


Theorem 4. If $\mathcal{R}_{0}>1$, then the system (3a-3j) has a unique endemic equilibrium $E_{1}$ in the interior of $\Omega_{1}$ and $E_{1}$ is asymptotically stable.

Using the Jacobian of the system $(3 \mathrm{a}-3 \mathrm{j})$ at the endemic equilibrium $E_{1}$, the proof of this theorem can be easily established following the method in Theorem 2 of this paper.

Theorem 5. If $R_{0}>1$, then the endemic equilibrium point, $E_{1}$, of the system (3a-3j) is unique and globally asymptotically stable (GAS) in the interior of $\Omega_{1}$.

Proof. The proof is based on the construction of a Lyapunov function (see $[22])$. Given the endemic equilibrium $E_{1}: \mathbf{x}=\mathbf{x}^{*}$, we let $D_{i}=x_{i}-x_{i}^{*}-x_{i}^{*} \ln \frac{x_{i}}{x_{i}^{*}}$ where $x_{i}$ represents each of the state variables of the system $(3 \mathrm{a}-3 \mathrm{j})$. The aim is to be obtain a Lyapunov function of the form $D=\sum_{i=1}^{n} c_{i} D_{i}$ where $D^{\prime} \leq 0$. We let $\mathbf{x}^{*} \equiv(1,2,3,4,5,6,7,8,9,10)$. Differentiating along the solutions, using (3a-3j) and the inequality $1-x+\ln x \leq 0, x>0$, with equality holding if and only if $x=1$ we get:

$$
\begin{aligned}
D_{1}^{\prime} & \leq B_{1 m} i_{1 m}^{*} s_{f}^{*}\left(\frac{i_{1 m}}{i_{1 m}^{*}}-\ln \frac{i_{1 m}}{i_{1 m}^{*}}-\frac{s_{f}^{*}}{s_{f}}+\ln \frac{s_{f}^{*}}{s_{f}}\right)+B_{2 m} i_{2 m}^{*} s_{f}^{*}\left(\frac{i_{2 m}}{i_{2 m}^{*}}\right. \\
& \left.-\ln \frac{i_{2 m}}{i_{2 m}^{*}}-\frac{s_{f}^{*}}{s_{f}}+\ln \frac{s_{f}^{*}}{s_{f}}\right)+B_{3 m} h_{m}^{*} s_{f}^{*}\left(\frac{h_{m}}{h_{m}^{*}}-\ln \frac{h_{m}}{h_{m}^{*}}-\frac{s_{f}^{*}}{s_{f}}\right. \\
& \left.+\ln \frac{s_{f}^{*}}{s_{f}}\right)+B_{4 m} a_{m}^{*} s_{f}^{*}\left(\frac{a_{m}}{a_{m}^{*}}-\ln \frac{a_{m}}{a_{m}^{*}}-\frac{s_{f}^{*}}{s_{f}}+\ln \frac{s_{f}^{*}}{s_{f}}\right) \\
& +\alpha_{f} a_{f} s_{f}\left(\frac{a_{f}^{*} s_{f}^{*^{2}}}{a_{f} s_{f}^{2}}-\ln \frac{a_{f}^{*} s_{f}^{*^{2}}}{a_{f} s_{f}^{2}}-\frac{s_{f}^{*}}{s_{f}}+\ln \frac{s_{f}^{*}}{s_{f}}\right) \\
& =a_{14} G_{14}+a_{16} G_{16}+a_{18} G_{18}+a_{110} G_{110}+a_{19} G_{19} \\
D_{2}^{\prime} & B_{1 f} i_{1 f}^{*} s_{m}^{*}\left(\frac{i_{1 f}}{i_{1 f}^{*}}-\ln \frac{i_{1 f}}{i_{1 f}^{*}}-\frac{s_{m}^{*}}{s_{m}}+\ln \frac{s_{m}^{*}}{s_{m}}\right)+B_{2 f} i_{2 f}^{*} s_{m}^{*}\left(\frac{i_{2 f}}{i_{2 f}^{*}}\right. \\
& \left.-\ln \frac{i_{2 f}}{i_{2 f}^{*}}-\frac{s_{m}^{*}}{s_{m}}+\ln \frac{s_{m}^{*}}{s_{m}}\right)+B_{3 f} h_{f}^{*} s_{m}^{*}\left(\frac{h_{f}}{h_{f}^{*}}-\ln \frac{h_{f}}{h_{f}^{*}}-\frac{s_{m}^{*}}{s_{m}}\right. \\
& \left.+\ln \frac{s_{m}^{*}}{s_{m}}\right)+B_{4 f} a_{f}^{*} s_{m}^{*}\left(\frac{a_{f}}{a_{f}^{*}}-\ln \frac{a_{f}}{a_{f}^{*}}-\frac{s_{m}^{*}}{s_{m}}+\ln \frac{s_{m}^{*}}{s_{m}}\right)
\end{aligned}
$$




$$
\begin{aligned}
+ & \alpha_{m} a_{m} s_{m}\left(\frac{a_{m}^{*} s_{m}^{*^{2}}}{a_{m} s_{m}^{2}}-\ln \frac{a_{m}^{*} s_{m}^{*^{2}}}{a_{m} s_{m}^{2}}-\frac{s_{m}^{*}}{s_{m}}+\ln \frac{s_{m}^{*}}{s_{m}}\right) \\
& =a_{23} G_{23}+a_{25} G_{25}+a_{27} G_{27}+a_{29} G_{29}+a_{210} G_{210} \\
D_{3}^{\prime} \leq & B_{1 m} i_{1 m} s_{f}\left(\frac{i_{1 f}^{*} i_{1 m}^{*} s_{f}^{*}}{i_{1 f} i_{1 m} s_{f}}-\ln \frac{i_{1 f}^{*} i_{1 m}^{*} s_{f}^{*}}{i_{1 f} i_{1 m} s_{f}}-\frac{i_{1 f}^{*}}{i_{1 f}}+\ln \frac{i_{1 f}^{*}}{i_{1 f}}\right) \\
+ & B_{2 m} i_{2 m} s_{f}\left(\frac{i_{1 f}^{*} i_{2 m}^{*} s_{f}^{*}}{i_{1 f} i_{2 m} s_{f}}-\ln \frac{i_{1 f}^{*} i_{2 m}^{*} s_{f}^{*}}{i_{1 f} i_{2 m} s_{f}}-\frac{i_{1 f}^{*}}{i_{1 f}}+\ln \frac{i_{1 f}^{*}}{i_{1 f}}\right) \\
+ & B_{3 m} h_{m} s_{f}\left(\frac{i_{1 f}^{*} h_{m}^{*} s_{f}^{*}}{i_{1 f} h_{m} s_{f}}-\ln \frac{i_{1 f}^{*} h_{m}^{*} s_{f}^{*}}{i_{1 f} h_{m} s_{f}}-\frac{i_{1 f}^{*}}{i_{1 f}}+\ln \frac{i_{1 f}^{*}}{i_{1 f}}\right) \\
+ & B_{4 m} a_{m} s_{f}\left(\frac{i_{1 f}^{*} a_{m}^{*} s_{f}^{*}}{i_{1 f} a_{m} s_{f}}-\ln \frac{i_{1 f}^{*} a_{m}^{*} s_{f}^{*}}{i_{1 f} a_{m} s_{f}}-\frac{i_{1 f}^{*}}{i_{1 f}}+\ln \frac{i_{1 f}^{*}}{i_{1 f}}\right) \\
+ & \alpha_{f} a_{f} i_{1 f}\left(\frac{a_{f}^{*} i_{1 f}^{*}}{a_{f} i_{1 f}^{2}}-\ln \frac{a_{f}^{*} i_{1 f}^{*}}{a_{f} i_{1 f}^{2}}-\frac{i_{1 f}^{*}}{i_{1 f}}+\ln \frac{i_{1 f}^{*}}{i_{1 f}}\right) \\
& =a_{34} G_{34}+a_{36} G_{36}+a_{38} G_{38}+a_{310} G_{310}+a_{39} G_{39}
\end{aligned}
$$

$$
\begin{aligned}
D_{4}^{\prime} & \leq B_{1 f} i_{1 f} s_{m}\left(\frac{i_{1 f}^{*} i_{1 m}^{*} s_{m}^{*}}{i_{1 f} i_{1 m} s_{m}}-\ln \frac{i_{1 f}^{*} i_{1 m}^{*} s_{m}^{*}}{i_{1 f} i_{1 m} s_{m}}-\frac{i_{1 m}^{*}}{i_{1 m}}+\ln \frac{i_{1 m}^{*}}{i_{1 m}}\right) \\
& +B_{2 f} i_{2 f} s_{m}\left(\frac{i_{2 f}^{*} i_{1 m}^{*} s_{m}^{*}}{i_{2 f} i_{1 m} s_{m}}-\ln \frac{i_{2 f}^{*} i_{1 m}^{*} s_{m}^{*}}{i_{2 f} i_{1 m} s_{m}}-\frac{i_{1 m}^{*}}{i_{1 m}}+\ln \frac{i_{1 m}^{*}}{i_{1 m}}\right) \\
& +B_{3 f} h_{f} s_{m}\left(\frac{h_{f}^{*} i_{1 m}^{*} s_{m}^{*}}{h_{f} i_{1 m} s_{m}}-\ln \frac{h_{f}^{*} i_{1 m}^{*} s_{m}^{*}}{h_{f} i_{1 m} s_{m}}-\frac{i_{1 m}^{*}}{i_{1 m}}+\ln \frac{i_{1 m}^{*}}{i_{1 m}}\right) \\
& +B_{4 f} a_{f} s_{m}\left(\frac{a_{f}^{*} i_{1 m}^{*} s_{m}^{*}}{a_{f} i_{1 m} s_{m}}-\ln \frac{a_{f}^{*} i_{1 m}^{*} s_{m}^{*}}{a_{f} i_{1 m} s_{m}}-\frac{i_{1 m}^{*}}{i_{1 m}}+\ln \frac{i_{1 m}^{*}}{i_{1 m}}\right) \\
& +\alpha_{m} a_{m} i_{1 m}\left(\frac{a_{m}^{*} i_{1 m}^{*}}{a_{m} i_{1 m}^{2}}-\ln \frac{a_{m}^{*} i_{1 m}^{*}}{a_{m} i_{1 m}^{2}}-\frac{i_{1 m}^{*}}{i_{1 m}}+\ln \frac{i_{1 m}^{*}}{i_{1 m}}\right) \\
D_{5}^{\prime} & \leq a_{43} G_{43}+a_{45} G_{45}+a_{47} G_{47}+a_{49} G_{49}+a_{410} G_{410} \\
& \left.-\ln \frac{i_{1 f}^{*} i_{2 f}^{*}}{i_{1 f} i_{2 f}}-\ln \frac{i_{1 f}^{*} i_{2 f}^{*}}{i_{1 f} i_{2 f}}-\frac{i_{2 f}^{*}}{i_{2 f}}+\ln \frac{i_{2 f}^{*}}{i_{2 f}}\right)+\alpha_{f f} a_{f} i_{2 f}\left(\frac{a_{f}^{*} i_{2 f}^{*}}{a_{f} i_{2 f}^{2}}\right. \\
i_{2 f} & \left.\ln \frac{i_{2 f}^{*}}{i_{2 f}}\right)=a_{53} G_{53}+a_{59} G_{59} \\
D_{6}^{\prime} & \leq \theta_{m} i_{1 m}\left(\frac{i_{1 m}^{*} i_{2 m}^{*}}{i_{1 m} i_{2 m}}-\ln \frac{i_{1 m}^{*} i_{2 m}^{*}}{i_{1 m} i_{2 m}}-\frac{i_{2 m}^{*}}{i_{2 m}}+\ln \frac{i_{2 m}^{*}}{i_{2 m}}\right)+\alpha_{m} a_{m} i_{2 m}
\end{aligned}
$$




$$
\begin{aligned}
& \left(\frac{a_{m}^{*} i_{2 m}^{*^{2}}}{a_{m} i_{2 m}^{2}} \ln \frac{a_{m}^{*} i_{2 m}^{*^{2}}}{a_{m} i_{2 m}^{2}}-\frac{i_{2 m}^{*}}{i_{2 m}}+\ln \frac{i_{2 m}^{*}}{i_{2 m}}\right)=a_{64} G_{64}+a_{610} G_{610} \\
& D_{7}^{\prime} \leq \pi_{f} i_{2 f}\left(\frac{i_{2 f}^{*} h_{f}^{*}}{i_{2 f} h_{f}}-\ln \frac{i_{2 f}^{*} h_{f}^{*}}{i_{2 f} h_{f}}-\frac{h_{f}^{*}}{h_{f}}+\ln \frac{h_{f}^{*}}{h_{f}}\right)+\alpha_{f} a_{f} h_{f}\left(\frac{a_{f}^{*} h_{f}^{*^{2}}}{a_{f} h_{f}^{2}}\right. \\
& \left.-\ln \frac{a_{f}^{*} h_{f}^{*^{2}}}{a_{f} h_{f}^{2}}-\frac{h_{f}^{*}}{h_{f}}+\ln \frac{h_{f}^{*}}{h_{f}}\right)=a_{75} G_{75}+a_{79} G_{79} \\
& D_{8}^{\prime} \leq \pi_{m} i_{2 m}\left(\frac{i_{2 m}^{*} h_{m}^{*}}{i_{2 m} h_{m}}-\ln \frac{i_{2 m}^{*} h_{m}^{*}}{i_{2 m} h_{m}}-\frac{h_{m}^{*}}{h_{m}}+\ln \frac{h_{m}^{*}}{h_{m}}\right)+\alpha_{m} a_{m} h_{m} \\
& \left(\frac{a_{m}^{*} h_{m}^{*^{2}}}{a_{m} h_{m}^{2}} \ln \frac{a_{m}^{*} h_{m}^{*^{2}}}{a_{m} h_{m}^{2}}-\frac{h_{m}^{*}}{h_{m}}+\ln \frac{h_{m}^{*}}{h_{m}}\right)=a_{86} G_{86}+a_{810} G_{810} \\
& D_{9}^{\prime} \leq \delta_{f} i_{1 f}\left(\frac{i_{1 f}^{*} a_{f}^{*}}{i_{1 f} a_{f}}-\ln \frac{i_{1 f}^{*} a_{f}^{*}}{i_{1 f} a_{f}}-\frac{a_{f}^{*}}{a_{f}}+\ln \frac{a_{f}^{*}}{a_{f}}\right)+\psi_{f} i_{2 f}\left(\frac{i_{2 f}^{*} a_{f}^{*}}{i_{2 f} a_{f}}\right. \\
& \left.-\ln \frac{i_{2 f}^{*} a_{f}^{*}}{i_{2 f} a_{f}}-\frac{a_{f}^{*}}{a_{f}}+\ln \frac{a_{f}^{*}}{a_{f}}\right)+\epsilon_{f} h_{f}\left(\frac{h_{f}^{*} a_{f}^{*}}{h_{f} a_{f}}-\ln \frac{h_{f}^{*} a_{f}^{*}}{h_{f} a_{f}}-\frac{a_{f}^{*}}{a_{f}}\right. \\
& \left.+\ln \frac{a_{f}^{*}}{a_{f}}\right)+a_{f}^{2}\left(\frac{a_{f}^{*^{3}}}{a_{f}^{3}}-\ln \frac{a_{f}^{*^{3}}}{a_{f}^{3}}-\frac{a_{f}^{*}}{a_{f}}+\ln \frac{a_{f}^{*}}{a_{f}}\right) \\
& =a_{93} G_{93}+a_{95} G_{95}+a_{97} G_{97}+a_{99} G_{99} \\
& D_{10}^{\prime} \leq \delta_{m} i_{1 m}\left(\frac{i_{1 m}^{*} a_{m}^{*}}{i_{1 m} a_{m}}-\ln \frac{i_{1 m}^{*} a_{m}^{*}}{i_{1 m} a_{m}}-\frac{a_{m}^{*}}{a_{m}}+\ln \frac{a_{m}^{*}}{a_{m}}\right) \\
& +\psi_{m} i_{2 m}\left(\frac{i_{2 m}^{*} a_{m}^{*}}{i_{2 m} a_{m}}-\ln \frac{i_{2 m}^{*} a_{m}^{*}}{i_{2 m} a_{m}}-\frac{a_{m}^{*}}{a_{m}}+\ln \frac{a_{m}^{*}}{a_{m}}\right) \epsilon_{m} h_{m}\left(\frac{h_{m}^{*} a_{m}^{*}}{h_{m} a_{m}}\right. \\
& \left.-\ln \frac{h_{m}^{*} a_{m}^{*}}{h_{m} a_{m}}-\frac{a_{m}^{*}}{a_{m}}+\ln \frac{a_{m}^{*}}{a_{m}}\right)+a_{m}^{2}\left(\frac{a_{m}^{*^{3}}}{a_{m}^{3}} \ln \frac{a_{m}^{*^{3}}}{a_{m}^{3}}-\frac{a_{m}^{*}}{a_{m}}+\ln \frac{a_{m}^{*}}{a_{m}}\right) \\
& =a_{104} G_{104}+a_{106} G_{106}+a_{108} G_{108}+a_{1010} G_{1010} \text {. }
\end{aligned}
$$

All other $a_{i j}=0$, and we ignore loops. Recalling the results in [22], values for $c_{i}, i=1, \ldots, 10$ are obtained by from Kirchhoff's theorem:

$$
\begin{aligned}
c_{1} & =a_{16}\left[a_{86}+a_{810}\left(a_{104}+a_{106}\right)\right]\left(a_{23}+a_{25}+a_{27}+a_{29}\right. \\
& \left.+a_{210}\right)\left(a_{53} a_{64} a_{75} a_{47}\right) \\
c_{2} & =a_{38}\left[a_{75}+a_{79}\left(a_{93}+a_{97}\right)\right]\left(a_{14}+a_{16}+a_{18}+a_{19}\right. \\
& \left.+a_{110}\right)\left(a_{49} a_{86} a_{610} a_{38}\right) \\
c_{3} & =a_{210} a_{64} a_{86} a_{108}\left(a_{14}+a_{16}+a_{18}+a_{19}+a_{110}\right)\left(a_{23}+a_{25}+a_{27}\right.
\end{aligned}
$$




$$
\begin{aligned}
& \left.+a_{29}+a_{210}\right) \\
c_{4} & =a_{19} a_{75} a_{95} a_{97}\left(a_{34}+a_{36}+a_{38}+a_{39}+a_{310}\right)\left(a_{43}+a_{45}+a_{47}\right. \\
& \left.+a_{49}+a_{410}\right) \\
c_{5} & =a_{86}\left(a_{16}+a_{18}+a_{110}+a_{108}\right)+a_{106}\left(a_{16}+a_{810}+a_{18}+a_{110}\right) \\
& +a_{16} a_{810} a_{104} \\
c_{6} & =a_{19} a_{53}\left(a_{75} a_{97}+a_{95}\right)\left(a_{59}+a_{75}+a_{97}+a_{95}\right)+a_{27}\left(a_{53}+a_{75}\right. \\
& \left.+a_{59}+a_{93}\right) \\
c_{7} & =a_{64} a_{104}\left(a_{18}+a_{610}+a_{108}+a_{106}\right)\left(a_{38}+a_{86} a_{310}\right) \\
& +a_{59} a_{43}\left(a_{49}+a_{95}\right) \\
c_{8} & =\left(a_{45}+a_{25}+a_{29}+a_{75}\right)\left(a_{49} a_{97}+a_{64}\right)+a_{36}\left(a_{64} a_{106}+a_{104}\right) \\
c_{9} & =a_{53} a_{75} a_{47}+\left(a_{34}+a_{810}\right)\left(a_{610}+a_{108}+a_{86}+a_{18}\right)+a_{14} a_{86} \\
c_{10} & =a_{25}\left(a_{59} a_{93}+a_{75} a_{79}\right)+\left(a_{75}+a_{27}+a_{95}+a_{53}\right)\left(a_{47} a_{79}\right) .
\end{aligned}
$$

Along each cycle: $G_{97}+G_{79}=0, G_{75}+G_{97}+G_{59}=0$,

$G_{108}+G_{810}=0, G_{86}+G_{108}+G_{610}=0, G_{104}+G_{410}=0$,

$G_{64}+G_{86}+G_{108}+G_{410}=0, G_{53}+G_{45}+G_{43}=0$,

$G_{95}+G_{59}=0, G_{310}+G_{34}+G_{104}=0, G_{49}+G_{43}+G_{93}=0$.

Therefore, the Lyapunov function is given by $D=\sum_{i=1}^{10} c_{i} D_{i}$. $D$ is a continuously differentiable function on the $\operatorname{int}\left(\Omega_{1}\right)$ and $D\left(E_{1}\right)=0$ where $E_{1}$ is the global minimum of $D$ on $\Omega_{1}$. The time derivative of $D$ computed along solutions of (3a)-(3j) is $D^{\prime} \leq 0$ for all nonnegative population states, where equality hold only at the endemic equilibrium point $E_{1}$ and $D$ is a Lyapunov function for system (3a)-(3j). Therefore, $E_{1}$ is a unique endemic equilibrium point of the model $(3 \mathrm{a})-(3 \mathrm{j})$. Thus, $E_{1}$ is unique and globally asymptotically stable in the $\operatorname{int}\left(\Omega_{1}\right)$ whenever $\mathcal{R}_{0}>1$.

\section{Analysis of optimal control}

We formulate an optimal control problem by introducing time dependent controls into the model $(3 \mathrm{a})-(3 \mathrm{j})$. The system to be controlled is defined as

$$
\left\{\begin{array}{cl}
\frac{d x}{d t}=f(x(t), u(t), t), & t_{0} \leq t \leq t_{\text {final }} \\
x\left(t_{0}\right)=x_{0}, & \text { given }
\end{array}\right.
$$


where the vector $x \in \mathbf{R}^{n}$ are the variables, that is, homogeneous sub-populations at each instant of time; $u \in \mathbf{R}^{p}$ are control functions of time; the vector $f(t, x, u):\left[t_{0}=0, t_{\text {final }}=T\right] \times \mathbf{R}^{n} \times \mathbf{R}^{p}$ and $\frac{d f}{d x}$ are continuous functions with respect to all their arguments; $n=10, p=8$. The control $u_{j 1}(t)(j=f, m)$ measures public health education and influences good sexual behaviour such as sticking to one partner while $u_{j 2}(t)$ represents the prevention control which reduces infectiousness of the HIV infectives. Examples of prevention methods include use of condoms, HIV prophylaxis, and voluntary male circumcision. $u_{j 3}(t)$ measures the rate of screening of unaware infectives at each time period while $u_{j 4}(t)$ measures the rate at which HIV infectives are treated. Each of the controls is bounded and may be a function of sex/health education and other factors. The model (3a)-(3j) becomes

$$
\begin{aligned}
\frac{d s_{f}}{d t} & =Q_{f}-\left(Q_{f}+u_{f 1}\right) s_{f}-\left(1-u_{f 2}\right) \beta_{m f} s_{f}+\alpha_{f} a_{f} s_{f} \\
\frac{d s_{m}}{d t} & =Q_{m}-\left(Q_{m}+u_{m 1}\right) s_{m}-\left(1-u_{m 2}\right) \beta_{f m} s_{m} \\
& +\alpha_{m} a_{m} s_{m} \\
\frac{d i_{1 f}}{d t} & =\left(1-u_{f 2}\right) \beta_{m f} s_{f}-\left(Q_{f}+\theta_{f}+\delta_{f}+u_{f 3}\right) i_{1 f} \\
& +\alpha_{f} a_{f} i_{1 f}, \\
\frac{d i_{1 m}}{d t} & =\left(1-u_{m 2}\right) \beta_{f m} s_{m}-\left(Q_{m}+\theta_{m}+\delta_{m}+u_{m 3}\right) i_{1 m} \\
& +\alpha_{m} a_{m} i_{1 m}, \\
\frac{d i_{2 f}}{d t} & =\left(\theta_{f}+u_{f 3}\right) i_{1 f}-\left(Q_{f}+\psi_{f}+\pi_{f}+u_{f 4}\right) i_{2 f} \\
& +\alpha_{f} a_{f} i_{2 f}, \\
\frac{d i_{2 m}}{d t} & =\left(\theta_{m}+u_{m 3}\right) i_{1 m}-\left(Q_{m}+\psi_{m}+\pi_{m}+u_{m 4}\right) i_{2 m} \\
& +\alpha_{m} a_{m} i_{2 m}, \\
\frac{d h_{f}}{d t} & =\left(\pi_{f}+u_{f 4}\right) i_{2 f}-\left(Q_{f}+\epsilon_{f}\right) h_{f}+\alpha_{f} h_{f} a_{f}, \\
\frac{d h_{m}}{d t} & =\left(\pi_{m}+u_{m 4}\right) i_{2 m}-\left(Q_{m}+\epsilon_{m}\right) h_{m}+\alpha_{m} h_{m} a_{m}, \\
\frac{d a_{f}}{d t} & =\delta_{f} i_{1 f}+\psi_{f} i_{2 f}+\epsilon_{f} h_{f}-\left(Q_{f}+\alpha_{f}\right) a_{f}+\alpha_{f} a_{f}^{2} \\
\frac{d a_{m}}{d t} & =\delta_{m} i_{1 m}+\psi_{m} i_{2 m}+\epsilon_{m} h_{m}-\left(Q_{m}+\alpha_{m}\right) a_{m}+\alpha_{m} a_{m}^{2}
\end{aligned}
$$


with non-negative known initial conditions $\mathbf{x}(0)$ and controls $0 \leq u_{j k} \leq 1$ for $j=f, m ; k=1,2,3,4$. Our goal is to seek optimal levels of the intervention strategies required to minimise the proportion of the susceptibles, unaware infectives, and the cost of applying the controls. We choose the objective functional $J$ defined as:

$$
\begin{aligned}
J & =\int_{0}^{T}\left[a_{1} s_{f}+a_{2} s_{m}+a_{3} i_{1 f}+a_{4} i_{1 m}+b_{1} u_{f 1}^{2}+b_{2} u_{m 1}^{2}\right. \\
& \left.+b_{3} u_{f 2}^{2}+b_{4} u_{m 2}^{2}+b_{5} u_{f 3}^{2}+b_{6} u_{m 3}^{2}+b_{7} u_{f 4}^{2}+b_{8} u_{m 4}^{2}\right] d t
\end{aligned}
$$

with $a_{1}, a_{2}, a_{3}, a_{4}$ being positive weight constants of the susceptibles and unaware infectives. The quantities $b_{k}, k=1, \cdots, 8$ are weight constants for the respective controls. The terms $b_{k} u_{j k}, j=f, m ; k=1,2,3,4$ describe the costs associated with public health education, prevention control (e.g., distribution of condoms), screening of unaware infectives and treatment of infectives. A quadratic functional is chosen by assuming nonlinear costs emanating from the control measures. We define the class of admissible controls by $U=\{\mathbf{u} \in$ $\left.L^{1}(0, T) \mid 0 \leq u_{j k}(t) \leq 1 \forall t \in[0, T]\right\}$ where $\mathbf{u}=\left(u_{f 1}, u_{m 1}, u_{f 2}, u_{m 2}, u_{f 3}, u_{m 3}, u_{f 4}\right.$, $\left.u_{m 4}\right)$. $U$ is assumed to consist of piecewise continuous controls. Our aim is to determine an optimal control set $\mathbf{u}^{\star}$ :

$$
J\left(\mathbf{u}^{\star}\right)=\min _{U}\{J(\mathbf{u})\} .
$$

\subsection{Existence of an optimal control set}

Theorem 6. There exists an optimal control $\mathbf{u}^{\star}$ and corresponding solution $\mathbf{x}^{\star}$ to the system $(9 a)-(9 j): J\left(\mathbf{u}^{\star}\right)=\min _{U}\{J(\mathbf{u})\}$.

Proof. We make reference to Theorem 4.1 and its accompanying Corollary 4.1 in Chapter III of [7]. We give the theorem's conditions below and later prove them:

1. The set of all solutions to the system (9a)-(9j) with corresponding controls in $U$ is non-empty;

2. The control set $U$ is closed;

3. The state system $(9 \mathrm{a})-(9 \mathrm{j})$ is linear in the control variables with coefficients depending on state variables and time; 
4. The integrand $L$ of the objective functional $J$ is convex in the controls;

5. $L(t, \mathbf{x}, \mathbf{u}) \geq c_{1}|\mathbf{u}| \beta-c_{2}$ where $c_{1}>0$ and $\beta>1$.

In order to verify condition 1, we use Picard-Lindelöf Theorem 3.1 on page 12 of [4]. If the state equations (9a)-(9j) are continuous and Lipschitz in the state variables and the corresponding solutions to the state equations are bounded, then there exist a unique solution corresponding to every admissible control in $U$. The total population proportions $n_{j}$ and their corresponding subpopulations for each sex are bounded below by zero and above by 1 . Therefore, the state system (9a)-(9j) is continuous and bounded. In order to guarantee uniqueness of the solution, we establish that the system (9a)-(9j) is Lipschitz regarding the state variables. This can be straightly obtained by showing that partial derivatives in the system $(9 \mathrm{a})-(9 \mathrm{j})$ with respect to the state variables exist and are bounded. Thus, condition 1 is proved. Clearly, our control set $U$ satisfies condition 2 . Condition 3 is proved by noticing that the RHS of the state system linearly depend on the controls. The integrand $L$ of $J$ is evidently convex on $U$ since $L$ is quadratic in the controls. Therefore, condition 4 is proved. To prove condition 3 , we note that

$$
\begin{aligned}
L & =a_{1} s_{f}+a_{2} s_{m}+a_{3} i_{1 f}+a_{4} i_{1 m}+b_{1} u_{f 1}^{2}+b_{2} u_{m 1}^{2}+b_{3} u_{f 2}^{2} \\
& +b_{4} u_{m 2}^{2}+b_{5} u_{f 3}^{2}+b_{6} u_{m 3}^{2}+b_{7} u_{f 4}^{2}+b_{8} u_{m 4}^{2} \\
& \geq b_{2} u_{m 1}^{2}+b_{3} u_{f 2}^{2}+b_{4} u_{m 2}^{2}+b_{5} u_{f 3}^{2}+b_{6} u_{m 3}^{2}+b_{7} u_{f 4}^{2}+b_{8} u_{m 4}^{2} \\
& \geq b_{1} u_{f 1}^{2}+b_{2} u_{m 1}^{2}+b_{3} u_{f 2}^{2}+b_{4} u_{m 2}^{2}+b_{5} u_{f 3}^{2}+b_{6} u_{m 3}^{2}+b_{7} u_{f 4}^{2} \\
& +b_{8} u_{m 4}^{2}-b_{1} \\
& \geq \min \left(b_{1}, b_{2}, b_{3}, b_{4}, b_{5}, b_{6}, b_{7}, b_{8}\right)\left(u_{f 1}^{2}+u_{m 1}^{2}+u_{f 2}^{2}+u_{m 2}^{2}+u_{f 3}^{2}\right. \\
& \left.+u_{m 3}^{2}+u_{f 4}^{2}+u_{m 4}^{2}\right)-b_{1}
\end{aligned}
$$

since $0 \leq u_{f 1} \leq 1$ implies that $\left(b_{1} u_{f 1}^{2}-b_{1}\right) \leq 0$. Therefore, there exists an optimal control pair $\left(\mathbf{x}^{\star}, \mathbf{u}^{\star}\right)$ which solves the optimal control problem (that is, minimise (10) subject to $(9 a)-(9 j))$.

\subsection{Characterisation of the optimal controls}

We apply Pontryagin's Maximum Principle (see [21]) to derive the necessary conditions for the optimal control pair, $(\mathbf{x}, \mathbf{u})$, whose existence was proved in Section 4.1. The system (9a)-(9j) and (11) are converted to a problem of minimising pointwise a non-linear $C^{2}$ function called the Hamiltonian $H(t, \mathbf{x}(t), \mathbf{u}(t), \boldsymbol{\Lambda}(t))$, 
with respect to $\mathbf{u}$ where

$$
\boldsymbol{\Lambda}=\left(\lambda_{1}(t), \lambda_{2}(t), \lambda_{3}(t), \lambda_{4}(t), \lambda_{5}(t), \lambda_{6}(t), \lambda_{7}(t), \lambda_{8}(t), \lambda_{9}(t), \lambda_{10}(t)\right)^{T} .
$$

Theorem 7. Given the optimal control pair $\left(\mathbf{x}^{\star}, \mathbf{u}^{\star}\right)$ that minimises the objective functional (10) subject to $(9 a)-(9 j)$, there exists a piecewise differentiable adjoint variable $\boldsymbol{\Lambda}(t)$, satisfying

$$
\begin{aligned}
& \frac{d \lambda_{1}}{d t}=-a_{1}+\left(1-u_{f 2}^{\star}\right) \beta_{m f}^{\star}\left(\lambda_{1}-\lambda_{3}\right)+\left(Q_{f}+u_{f 1}^{\star}-\alpha_{f} a_{f}^{\star}\right) \lambda_{1} \\
& \frac{d \lambda_{2}}{d t}=-a_{2}+\left(1-u_{m 2}^{\star}\right) \beta_{f m}^{\star}\left(\lambda_{2}-\lambda_{4}\right)+\left(Q_{m}+u_{m 1}^{\star}-\alpha_{m} a_{m}^{\star}\right) \lambda_{2} \\
& \frac{d \lambda_{3}}{d t}=-a_{3}+\left(1-u_{m 2}^{\star}\right) s_{m}^{\star} B_{1 f}^{\star}\left(\lambda_{2}-\lambda_{4}\right)+\left(\theta_{f}+u_{f 3}^{\star}\right)\left(\lambda_{3}-\lambda_{5}\right) \\
& +\delta_{f}\left(\lambda_{3}-\lambda_{9}\right)+\left(Q_{f}-\alpha_{f} a_{f}^{\star}\right) \lambda_{3} \\
& \frac{d \lambda_{4}}{d t}=-a_{4}+\left(1-u_{f 2}^{\star}\right) s_{f}^{\star} B_{1 m}^{\star}\left(\lambda_{3}-\lambda_{5}\right)+\left(\theta_{m}+u_{m 3}^{\star}\right)\left(\lambda_{4}-\lambda_{6}\right) \\
& +\delta_{m}\left(\lambda_{4}-\lambda_{10}\right)+\left(Q_{m}-\alpha_{m} a_{m}^{\star}\right) \lambda_{4} \\
& \frac{d \lambda_{5}}{d t}=\left(1-u_{m 2}^{\star}\right) s_{m}^{\star} B_{2 f}^{\star}\left(\lambda_{2}-\lambda_{4}\right)+\left(\pi_{f}+u_{f 4}^{\star}\right)\left(\lambda_{5}-\lambda_{7}\right) \\
& +\psi_{f}\left(\lambda_{5}-\lambda_{9}\right)+\left(Q_{f}-\alpha_{f} a_{f}^{\star}\right) \lambda_{5} \\
& \frac{d \lambda_{6}}{d t}=\left(1-u_{f 2}^{\star}\right) s_{f}^{\star} B_{2 m}^{\star}\left(\lambda_{3}-\lambda_{5}\right)+\left(\pi_{m}+u_{m 4}^{\star}\right)\left(\lambda_{6}-\lambda_{8}\right) \\
& +\psi_{m}\left(\lambda_{6}-\lambda_{10}\right)+\left(Q_{m}-\alpha_{m} a_{m}^{\star}\right) \lambda_{6} \\
& \frac{d \lambda_{7}}{d t}=\left(1-u_{m 2}^{\star}\right) s_{m}^{\star} B_{3 f}^{\star}\left(\lambda_{2}-\lambda_{4}\right)+\epsilon_{f}\left(\lambda_{7}-\lambda_{9}\right) \\
& +\left(Q_{f}-\alpha_{f} a_{f}^{\star}\right) \lambda_{7} \\
& \frac{d \lambda_{8}}{d t}=\left(1-u_{f 2}^{\star}\right) s_{f}^{\star} B_{3 m}^{\star}\left(\lambda_{3}-\lambda_{5}\right)+\epsilon_{m}\left(\lambda_{8}-\lambda_{10}\right) \\
& +\left(Q_{m}-\alpha_{m} a_{m}^{\star}\right) \lambda_{8} \\
& \frac{d \lambda_{9}}{d t}=-\alpha_{f}\left(s_{f}^{\star} \lambda_{1}+i_{1 f}^{\star} \lambda_{3}+i_{2 f}^{\star} \lambda_{5}+h_{f}^{\star} \lambda_{7}\right) \\
& +\left(1-u_{m 2}^{\star}\right) s_{m}^{\star} B_{4 f}^{\star}\left(\lambda_{2}-\lambda_{4}\right)+\left(Q_{f}+\alpha_{f}-2 \alpha_{f} a_{f}^{\star}\right) \lambda_{9} \\
& \frac{d \lambda_{10}}{d t}=-\alpha_{m}\left(s_{m}^{\star} \lambda_{2}+i_{1 m}^{\star} \lambda_{4}+i_{2 m}^{\star} \lambda_{6}+h_{m}^{\star} \lambda_{8}\right) \\
& +\left(1-u_{f 2}^{\star}\right) s_{f}^{\star} B_{4 m}^{\star}\left(\lambda_{3}-\lambda_{5}\right)+\left(Q_{m}+\alpha_{m}-2 \alpha_{m} a_{m}^{\star}\right) \lambda_{10}
\end{aligned}
$$

with transversality conditions $\lambda_{i}(T)=0 \forall i=1, \cdots, 10$. Moreover, the optimal 
control set is characterised by the continuous functions

$$
\begin{aligned}
& u_{f 1}^{\star}=\min \left\{1, \max \left\{0, \frac{s_{f}^{\star}}{2 b_{1}} \lambda_{1}\right\}\right\} \\
& u_{m 1}^{\star}=\min \left\{1, \max \left\{0, \frac{s_{m}^{\star}}{2 b_{2}} \lambda_{2}\right\}\right\} \\
& u_{f 2}^{\star}=\min \left\{1, \max \left\{0, \frac{\beta_{m f}^{\star} s_{f}^{\star}}{2 b_{3}}\left(\lambda_{3}-\lambda_{1}\right)\right\}\right\} \\
& u_{m 2}^{\star}=\min \left\{1, \max \left\{0, \frac{\beta_{f m}^{\star} s_{m}^{\star}}{2 b_{4}}\left(\lambda_{4}-\lambda_{2}\right)\right\}\right\} \\
& u_{f 3}^{\star}=\min \left\{1, \max \left\{0, \frac{i_{1 f}^{\star}}{2 b_{5}}\left(\lambda_{3}-\lambda_{5}\right)\right\}\right\} \\
& u_{m 3}^{\star}=\min \left\{1, \max \left\{0, \frac{i_{1 m}^{\star}}{2 b_{6}}\left(\lambda_{4}-\lambda_{6}\right)\right\}\right\} \\
& u_{f 4}^{\star}=\min \left\{1, \max \left\{0, \frac{i_{2 f}^{\star}}{2 b_{7}}\left(\lambda_{5}-\lambda_{7}\right)\right\}\right\} \\
& u_{m 4}^{\star}=\min \left\{1, \max \left\{0, \frac{i_{2 m}^{\star}}{2 b_{8}}\left(\lambda_{6}-\lambda_{8}\right)\right\}\right\} .
\end{aligned}
$$

Proof. We systematically apply Pontryagin's Maximum Principle (see [13],[21]) to obtain the required necessary conditions. The Hamiltonian, $H$, is given by

$$
\begin{aligned}
H & =a_{1} s_{f}+a_{2} s_{m}+a_{3} i_{1 f}+a_{4} i_{1 m}+b_{1} u_{f 1}^{2}+b_{2} u_{m 1}^{2}+b_{3} u_{f 2}^{2} \\
& +b_{4} u_{m 2}^{2}+b_{5} u_{f 3}^{2}+b_{6} u_{m 3}^{2}+b_{7} u_{f 4}^{2}+b_{8} u_{m 4}^{2}+\lambda_{1}\left[Q_{f}-\left(Q_{f}\right.\right. \\
& \left.\left.+u_{f 1}\right) s_{f}-\left(1-u_{f 2}\right) \beta_{m f} s_{f}+\alpha_{f} a_{f} s_{f}\right]+\lambda_{2}\left[Q_{m}-\left(Q_{m}\right.\right. \\
& \left.\left.+u_{m 1}\right) s_{m}-\left(1-u_{m 2}\right) \beta_{f m} s_{m}+\alpha_{m} a_{m} s_{m}\right]+\lambda_{3}\left[\left(1-u_{f 2}\right) \beta_{m f} s_{f}\right. \\
- & \left.\left(Q_{f}+\theta_{f}+\delta_{f}+u_{f 3}\right) i_{1 f}+\alpha_{f} a_{f} i_{1 f}\right]+\lambda_{4}\left[\left(1-u_{m 2}\right) \beta_{f m} s_{m}\right. \\
- & \left.\left(Q_{m}+\theta_{m}+\delta_{m}+u_{m 3}\right) i_{1 m}+\alpha_{m} a_{m} i_{1 m}\right]+\lambda_{5}\left[\left(\theta_{f}+u_{f 3}\right) i_{1 f}\right. \\
- & \left.\left(Q_{f}+\psi_{f}+\pi_{f}+u_{f 4}\right) i_{2 f}+\alpha_{f} a_{f} i_{2 f}\right]+\lambda_{6}\left[\left(\theta_{m}+u_{m 3}\right) i_{1 m}\right. \\
- & \left.\left(Q_{m}+\psi_{m}+\pi_{m}+u_{m 4}\right) i_{2 m}+\alpha_{m} a_{m} i_{2 m}\right]+\lambda_{7}\left[\left(\pi_{f}+u_{f 4}\right) i_{2 f}\right. \\
- & \left.\left(Q_{f}+\epsilon_{f}\right) h_{f}+\alpha_{f} h_{f} a_{f}\right]+\lambda_{8}\left[\left(\pi_{m}+u_{m 4}\right) i_{2 m}-\left(Q_{m}+\epsilon_{m}\right) h_{m}\right. \\
+ & \left.\alpha_{m} h_{m} a_{m}\right]+\lambda_{9}\left[\delta_{f} i_{1 f}+\psi_{f} i_{2 f}+\epsilon_{f} h_{f}-\left(Q_{f}+\alpha_{f}\right) a_{f}+\alpha_{f} a_{f}^{2}\right] \\
+ & \lambda_{10}\left[\delta_{m} i_{1 m}+\psi_{m} i_{2 m}+\epsilon_{m} h_{m}-\left(Q_{m}+\alpha_{m}\right) a_{m}+\alpha_{m} a_{m}^{2}\right] .
\end{aligned}
$$

We use the equations $\frac{d \lambda_{j}}{d t}=-\frac{\partial H}{\partial x_{j}}$ to get the adjoint variables and they must 
satisfy the transversality conditions $\lambda_{j}=0 \forall i=1,2, \cdots, 10$. We apply the optimality equation $\frac{\partial H}{\partial u_{j k}^{\star}}=0$ for all values $j=f, m ; k=1,2,3,4$ in order to find characterisation of the optimal control $\mathbf{u}^{\star}$ in terms of $\mathbf{x}^{\star}$ and $\boldsymbol{\Lambda}$ :

$$
\begin{aligned}
& \frac{\partial H}{\partial u_{f 1}^{\star}}=2 b_{1} u_{f 1}^{\star}-s_{f}^{\star} \lambda_{1} ; \quad \frac{\partial H}{\partial u_{m 1}^{\star}}=2 b_{2} u_{m 1}^{\star}-s_{m}^{\star} \lambda_{2} ; \\
& \frac{\partial H}{\partial u_{f 2}^{\star}}=2 b_{3} u_{f 2}^{\star}+\beta_{m f}^{\star} s_{f}^{\star}\left(\lambda_{1}-\lambda_{3}\right) ; \quad \frac{\partial H}{\partial u_{m 2}^{\star}}=2 b_{4} u_{m 2}^{\star}+\beta_{f m}^{\star} s_{m}^{\star}\left(\lambda_{2}-\lambda_{4}\right) ; \\
& \frac{\partial H}{\partial u_{f 3}^{\star}}=2 b_{5} u_{f 3}^{\star}+i_{1 f}^{\star}\left(\lambda_{5}-\lambda_{3}\right) ; \quad \frac{\partial H}{\partial u_{m 3}^{\star}}=2 b_{6} u_{m 3}^{\star}+i_{1 m}^{\star}\left(\lambda_{6}-\lambda_{4}\right) ; \\
& \frac{\partial H}{\partial u_{f 4}^{\star}}=2 b_{7} u_{f 4}^{\star}+i_{2 f}^{\star}\left(\lambda_{7}-\lambda_{5}\right) ; \quad \frac{\partial H}{\partial u_{m 4}^{\star}}=2 b_{8} u_{m 4}^{\star}+i_{2 m}^{\star}\left(\lambda_{8}-\lambda_{6}\right) .
\end{aligned}
$$

We obtain:

$$
\begin{aligned}
& u_{f 1}^{\star}=\frac{s_{f}^{\star}}{2 b_{1}} \lambda_{1} ; \quad u_{m 1}^{\star}=\frac{s_{m}^{\star}}{2 b_{2}} \lambda_{2} ; \quad u_{f 2}^{\star}=\frac{\beta_{m f}^{\star} s_{f}^{\star}}{2 b_{3}}\left(\lambda_{3}-\lambda_{1}\right) ; \\
& u_{m 2}^{\star}=\frac{\beta_{f m}^{\star} s_{m}^{\star}}{2 b_{4}}\left(\lambda_{4}-\lambda_{2}\right) ; \quad u_{f 3}^{\star}=\frac{i_{1 f}^{\star}}{2 b_{5}}\left(\lambda_{3}-\lambda_{5}\right) ; \quad u_{m 3}^{\star}=\frac{i_{1 m}^{\star}}{2 b_{6}}\left(\lambda_{4}-\lambda_{6}\right) ; \\
& u_{f 4}^{\star}=\frac{i_{2 f}^{\star}}{2 b_{7}}\left(\lambda_{5}-\lambda_{7}\right) ; \quad u_{m 4}^{\star}=\frac{i_{2 m}^{\star}}{2 b_{8}}\left(\lambda_{6}-\lambda_{8}\right) .
\end{aligned}
$$

Applying the bounds on the controls $u_{j}^{\star}=\left\{\begin{array}{cc}u_{j} & \text { if } 0<u_{1}<1 \\ 0 & \text { if } u_{j} \leq 0 \\ 1 & \text { if } u_{j} \geq 1\end{array}\right.$, we obtain the required characterisation. Therefore Theorem 7 is proved.

\section{Numerical results}

Numerical simulations to approximate the optimal controls are carried out using the Forward-Backward Sweep method whose algorithm is described on page 50 in [13]. The optimality system consists of twenty ordinary differential equations having 10 state equations and 10 adjoint equations. State equations have initial conditions and adjoint equations have boundary conditions at the final time $T$. Then simultaneously, state space variables are computed forward in time and the adjoints (or costates) are computed backward in time in the same interval together with the solution of the state variables transversality conditions. Adjoint equations are computed backward in time since transversality 
conditions are final time conditions. We provide an initial guess for the controls for the process to start and the controls are continuously updated using state and adjoint values with the characterisation in (12). We adjust the initial guess in instances where convergence problems are encountered. The whole process is repeated until convergence occurs, that is, when current state, adjoint and control values are sufficiently close to successive values. We plot states in the absence of controls and in the presence of controls to determine the best intervention strategies.

We use different initial conditions and weight constants $a_{1}, a_{2}, a_{3}, a_{4} ; b_{i}, i=1, \cdots, 8$ to obtain several control strategies for a 50 -year plan. Figures (1)-(6) were obtained using $\mathbf{x}(0)=(0.860,0.800,0.057,0.100,0.029,0.030,0.036,0.040,0.018$, $0.030)$ and weight constants $a_{1}=7, a_{2}=5, a_{3}=2, a_{4}=3$ and $b_{1}=14, b_{2}=$ $12, b_{3}=28, b_{4}=24, b_{5}=6, b_{6}=7, b_{7}=52, b_{8}=48$. The weight constants are assumed to be proportional to the cost of the controls. The other parameters are shown in Table 2.

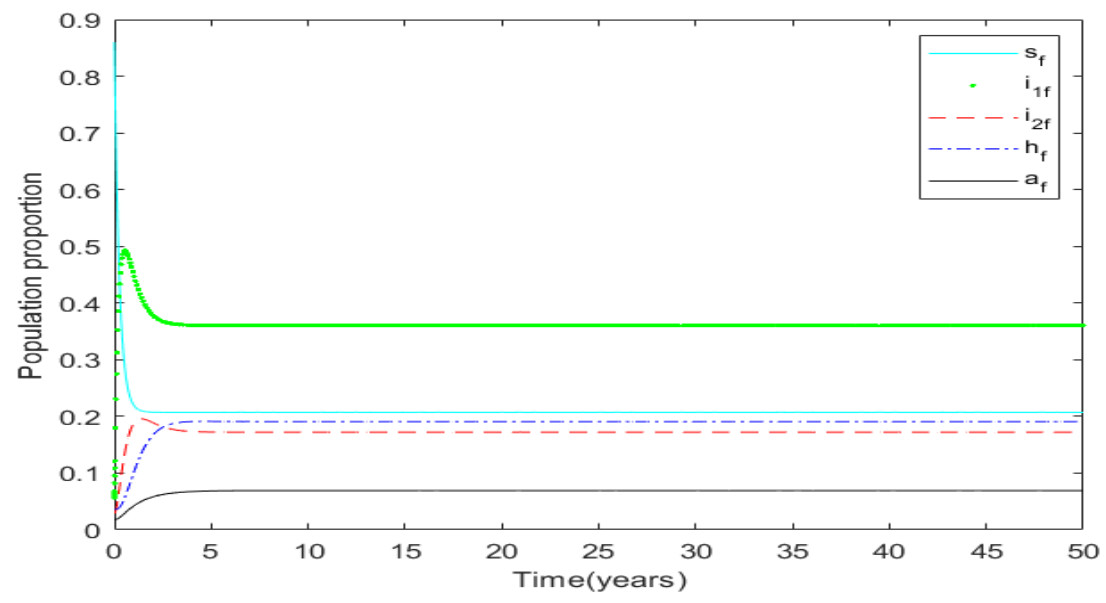

Figure 1: Variation of female population without controls

When no controls are administered, unaware infectives rise from below $10 \%$ of the population and level around $40 \%$ of the population in about two years as shown in Figures 1 and 2. However, in the presence of controls, Figure 3 shows that the unaware infectives reach their peak within one year and falls to about $10 \%$ of the population within the next four years. In the case where all controls are fully administered, the susceptible population immediately starts to decrease to about $10 \%$ of the population in the first two years 
Table 2: Parameter values used: The assumed values are based on HIV/AIDS dynamics of Zimbabwe, one of the Southern African countries

\begin{tabular}{llll}
\hline Symbol & Female & Male & Source \\
\hline$\beta_{I_{1 i}}$ & 0.28 & 0.42 & see $([1],[29])$ \\
$\beta_{I_{2 i}}$ & 0.18 & 0.24 & see $([1],[29])$ \\
$\beta_{H_{i}}$ & 0.14 & 0.16 & see $([1],[29])$ \\
$\beta_{A_{i}}$ & 0.10 & 0.12 & see $([1],[29])$ \\
$Q_{i}$ & 0.016 & 0.012 & Assumed \\
$c_{I_{1 i}}$ & 4 & 6 & see $([3],[12])$ \\
$c_{I_{2 i}}$ & 3 & 4 & see $([3],[12])$ \\
$c_{H_{i}}$ & 2 & 3 & see $([3],[12])$ \\
$c_{A_{i}}$ & 1 & 2 & see $([3],[12])$ \\
$b_{I_{1 i}}$ & 0.7 & 0.8 & Assumed \\
$b_{I_{2 i}}$ & 0.65 & 0.75 & Assumed \\
$b_{H_{i}}$ & 0.5 & 0.6 & Assumed \\
$b_{A_{i}}$ & 0.3 & 0.4 & Assumed \\
$\theta_{i}$ & 0.8 & 0.69 & see $([17],[33])$ \\
$\delta_{i}$ & 0.24 & 0.20 & see $([31])$ \\
$\psi_{i}$ & 0.16 & 0.12 & see $([1],[24],[31])$ \\
$\pi_{i}$ & 0.62 & 0.51 & see $([17])$ \\
$\epsilon_{i}$ & 0.0018 & 0.0011 & see $([31])$ \\
$\alpha_{j}$ & 0.025 & 0.021 & see $([30],[33])$ \\
$\alpha_{j}$ & 0.025 & 0.021 & see $([30],[33])$ \\
$\mu_{i}$ & 0.02 & 0.018 & see $([17],[33])$ \\
\hline & & &
\end{tabular}




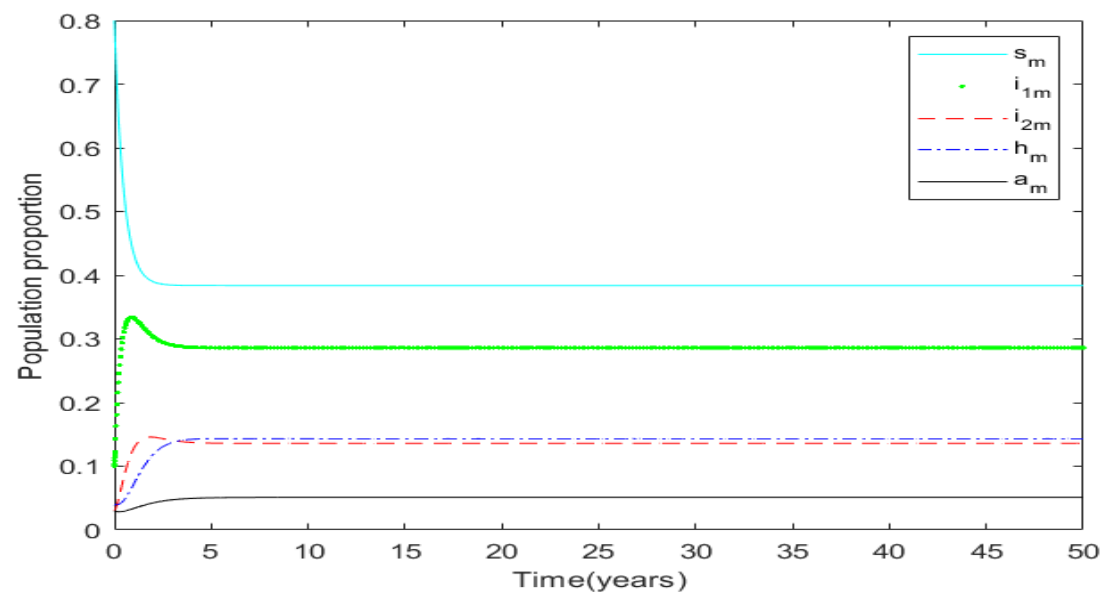

Figure 2: Variation of male population without controls
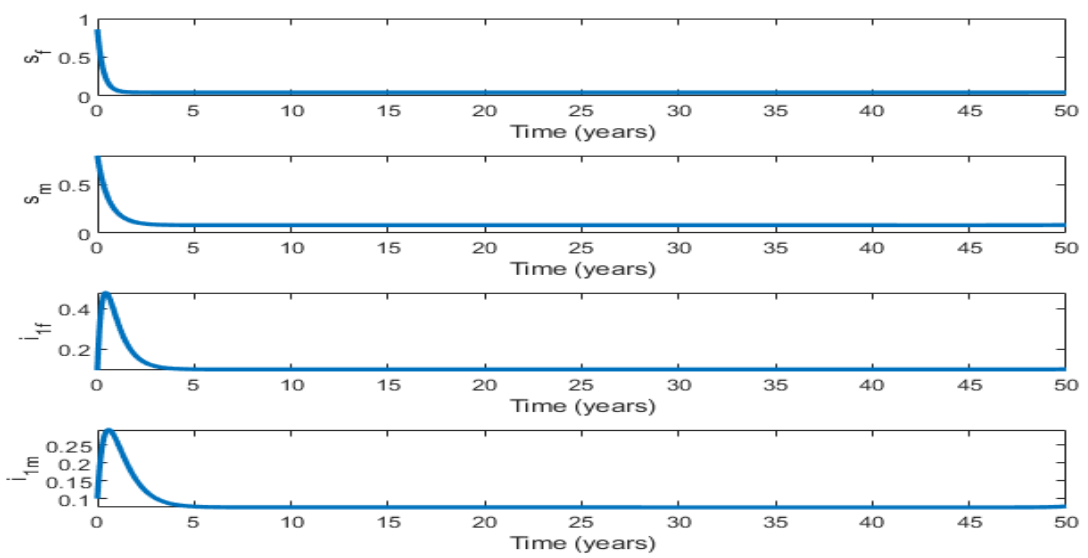

Figure 3: Variation of population $\left(s_{f}, s_{m}, i_{1 f}, i_{1 m}\right)$ when all controls are applied 

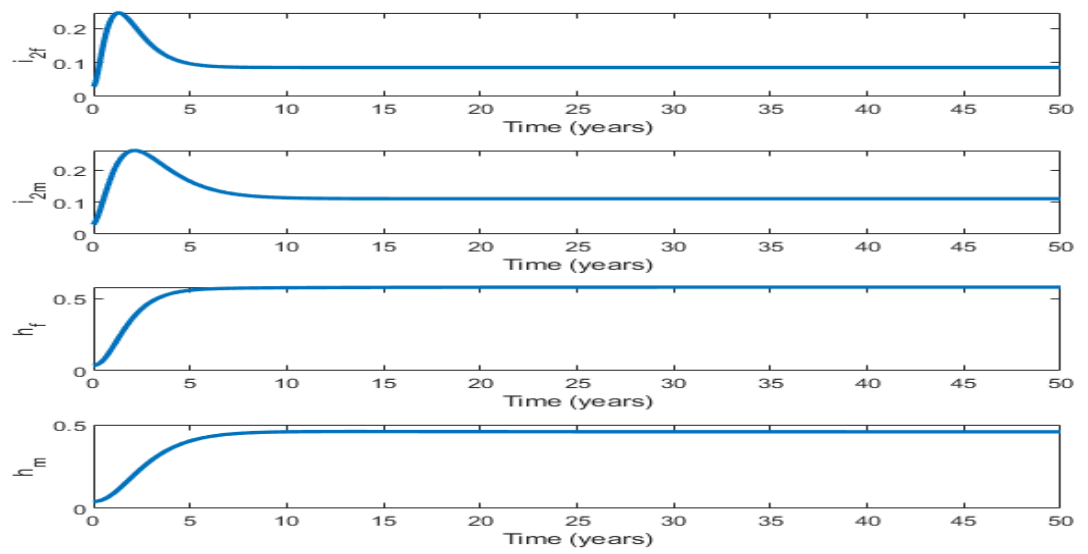

Figure 4: Variation of population $\left(i_{2 f}, i_{2 m}, h_{f}, h_{m}\right)$ when all controls are applied
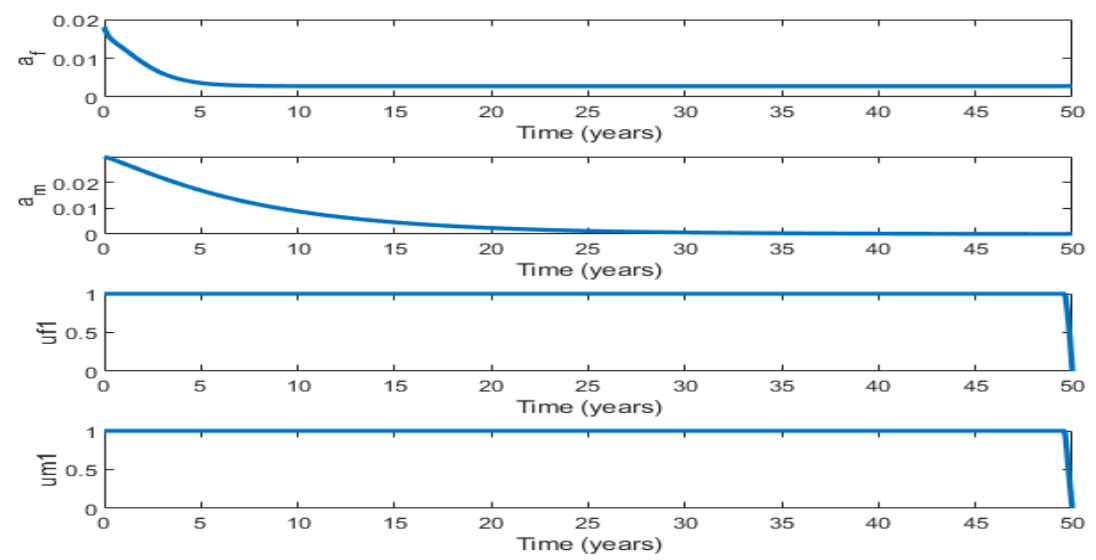

Figure 5: Variation of population $\left(a_{f}, a_{m}\right)$ and optimal controls $u_{f 1}, u_{m 1}$ when all controls are applied 

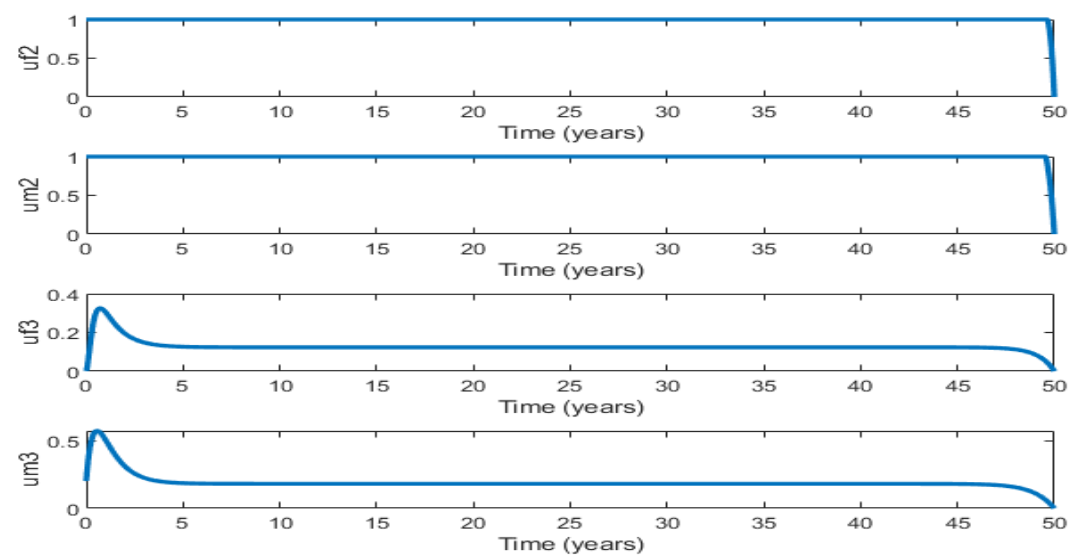

Figure 6: Profiles for controls $u_{f 2}, u_{m 2}, u_{f 3}$ and $u_{m 3}$ when all controls are applied. Profiles for controls $u_{f 4}$ and $u_{m 4}$ are not shown since they are similar to those for $u_{f 2}$ and $u_{m 2}$

and fewer females than males are susceptible (see Figure 3). Control profiles for $u_{f 1}, u_{m 1}, u_{f 2}, u_{m 2}, u_{f 4}$ and $u_{m 4}$ are similar. The sex education $\left(u_{f 1}, u_{m 1}\right)$ and HIV prevention $\left(u_{f 2}, u_{m}\right)$ controls reduce the susceptible population, while the screening control $\left(u_{f 3}, u_{m 3}\right)$ decreases the unaware infective population, thereby minimising HIV transmission. Moreover, the screening control gives rise to the aware infectives which are then recruited for treatment. The combination of all controls is responsible for the reduction of AIDS individuals to almost zero, that is, the effect of appropriate education, prevention, screening and treatment.

\section{Conclusion}

A heterosexual HIV/AIDS model is formulated and analysed, especially its wellposedness, existence of a disease threshold parameter and asymptotic stability of disease free and endemic equilibrium points. Apart from being a variable population size model, our model assumes asymmetry in female and male HIV transmission and progression rates, a feature that is not evident in models in literature, to the best of our knowledge. Optimal control theories are applied to establish the existence of an optimal control solution and its characterisation. We numerically solve the optimal control problem (since such problems are difficulty to solve analytically) using the forward-backward sweep method 
based on Runge Kutta fourth order scheme. Administering in full scale, all the four controls (sex education, HIV prevention methods, screening, treatment) produces the optimal control plan in which susceptibles and unaware infectives are reduced to below $10 \%$ of the population while all screened infectives are recruited for treatment, thereby greatly reducing the epidemic. In practice, HIV/AIDS dynamics is more complicated than as depicted in this model. However, our study demonstrates the benefits of mathematical modelling in reducing the HIV/AIDS disease.

\section{References}

[1] J.M. Baeten, J. Overbaugh, Measuring the infectiousness of persons with HIV-1: opportunities for preventing sexual HIV-1 transmission, Current HIV Research, 1, No 1 (2003), 69-86.

[2] S. Busenberg, P. van den Driessche, Analysis of a Disease Transmission model in a population with varying size, Journal of Mathematical Biology, 28, No 3 (1990), 257-270.

[3] M. Chopra, L. Townsend, L. Johnston, et al., Estimating HIV prevalence and risk behaviors among high-risk heterosexual men with multiple sex partners: Use of respondent-driven sampling, Journal of Acquired Immune Deficiency Syndrome, 51 (2009), 72-77.

[4] E.A. Coddington, N. Levinson, Theory of Ordinary Differential Equations, TMH Edition, Tata McGraw-Hill Publ. Co. Ltd, New Delhi (1972).

[5] O. Diekmann, J. A. Heesterbeek, J.A. Metz, On the definition and the computation of the basic reproduction ratio $\mathrm{R} 0$ in models for infectious diseases in heterogeneous populations, Journal of Mathematical Biology, 28, No 4 (1990), 365-382.

[6] L. Esteva, C. Vargas, A model for dengue disease with variable human population, Journal of Mathematical Biology, 38, No 3 (1999), 220-240.

[7] W.H. Fleming, R.W. Rishel, Deterministic and stochastic optimal control, 1st Ed., Springer-Verlag, Berlin-Heidelberg-New York (1975).

[8] H. Gaff, E. Schaefer, Optimal control applied to vaccination and treatment strategies for various epidemiological models, Mathematical Biosciences and Engineering, 6, No 3 (2009), 469-492. 
[9] H.W. Hethcote, The mathematics of infectious diseases, SIAM Review 42, No 4 (2000), 599-653.

[10] J.L. Juusola, M.L. Brandeau, HIV Treatment and Prevention: A simple model to determine optimal investment, Medical Decision Making, 36, No 3 (2016), 391-409.

[11] W. Kermack, A. McKendrick, A contribution to the mathematical theory of epidemics, Proc. of the Royal Society of London. Ser. A, Containing Papers of a Mathematical and Physical Character, 115, No 772 (1927), 700-721.

[12] J.H. Kim, HIV transmissions by stage and sex role in long-term concurrent sexual partnerships, Acta Biotheoretica, 63 (2015), 33-54.

[13] J. Lenhart, S. and Workman, Optimal Control Applied to Biological Models, Chapman Hall/CRC, Boca Raton-London-New York (2007).

[14] M.Y. Li, J.R. Graef, L. Wang, Global dynamics of a SEIR model with a varying total population size, Mathematical Biosciences, 160, No 2 (1999), 191-213.

[15] A. Mallela, S. Lenhart, N.K. Vaidya, HIV/TB co-infection treatment: Modeling and optimal control theory perspectives, Journal of Computational and Applied Mathematics, 307 (2015), 143-161.

[16] C.C. Mccluskey, A model of HIV / AIDS with staged progression and amelioration, Mathematical Biosciences, 181 (2003), 1-16.

[17] Ministry of Health and Child Care Zimbabwe AIDS and TB Unit, Zimbabwe HIV Drug Resistance Early Warning Indicators (EWI) Survey: 2013 Report, Technical Report, Harare, (2013).

[18] R.E. Miron, R.J. Smith, Resistance to protease inhibitors in a model of HIV-1 INFECTION WITH IMPULSIVE DRUG EFFECTS, Bull. of Mathematical Biology, 76 No 1 (2014), 59-97.

[19] G.A. Ngwa, W.S. Shu, A mathematical model for endemic malaria with variable human and mosquito populations, Mathematical and Computer Modelling, 32, No 7-8 (2000), 747-763.

[20] C. Orrell, Antiretroviral adherence in a resource-poor setting, Current HIV/AIDS Reports, 2, No 4 (2005), 171-176. 
[21] L. Pontryagin, V. Boltyanskii, R. Gamkrelidze, E. Mishchenko, The Mathematical Theory of Optimal Processes, Interscience Publishers, New YorkLondon (1962).

[22] Z. Shuai, P. van den Driessche, Global stability of infectious disease models using Lyapunov functions, SIAM Journal on Applied Mathematics, 73, No 4 (2013), 1513-1532.

[23] R.F. Stengel, Mutation and control of the human immunodeficiency virus, Mathematical Biosciences 213, No 2 (2008), 93-102.

[24] T. Sterling, D. Vlahov, et al., Initial PLASMA HIV-1 RNA levels and progression to AIDS in women and men, New England Journal of Medicine, 344 (2001), 720-726.

[25] UNAIDS, Global AIDS Update, Techn. Report, Geneva (2016).

[26] UNAIDS, Fact Sheet, N. Hiv, 2017 Global HIV Statistics, Techn. Report, July (2018).

[27] UNAIDS, GLOBAL REPORT: UNAIDS Report on the global AIDS epidemic (2013).

[28] P. van den Driessche, J. Watmough, Reproduction numbers and subthreshold endemic equilibria for compartmental models of disease transmission, Mathematical Biosciences, 180, No 1-2 (2002), 29-48.

[29] B. Varghese, J.E. Maher, etal, Reducing the risk of sexual HIV transmission: quantifying the per-act risk for HIV on the basis of choice of partner, sex act, and condom use, Sexually Transmitted Diseases, 29 (2002), 38-43.

[30] WHO, Number of deaths due to HIV/AIDS by country, Technical Report (2017).

[31] K.H. Wong, K.C. Chan, S.S. Lee, Delayed progression to death and to AIDS in a Hong Kong cohort of patients with advanced HIV type 1 disease during the era of highly active antiretroviral therapy, Clinical Infectious Diseases, 39 (2004), 853-860.

[32] Y. Zhou, Y. Liang, J. Wu, An optimal strategy for HIV multitherapy, Journal of Computational and Applied Mathematics, 263 (2014), 326-337.

[33] ZIMSTAT, ICF International, Demographic and Health Survey Key Findings, Technical Report (2016). 
